\title{
CONSTRUCTION OF CENTRAL ELEMENTS IN THE AFFINE HECKE ALGEBRA VIA NEARBY CYCLES
}

\author{
D. GAITSGORY
}

\section{INTRODUCTION}

0.1. Overview. Let $G$ be a connected reductive group over a finite field $\mathbb{F}_{q}$ and let $G(\widehat{\mathcal{K}})$ be the corresponding group over the local field $\widehat{\mathcal{K}}=\mathbb{F}_{q}((t))$. Let $G(\widehat{\mathcal{O}}) \subset G(\widehat{\mathcal{K}})$ be a maximal compact subgroup of $G(\widehat{\mathcal{K}})$ (here $\left.\widehat{\mathcal{O}}=\mathbb{F}_{q}[[t]]\right)$ and let $\mathbf{H}_{\text {sph }}$ denote the Hecke algebra of $G(\widehat{\mathcal{K}})$ with respect to $G(\widehat{\mathcal{O}})$.

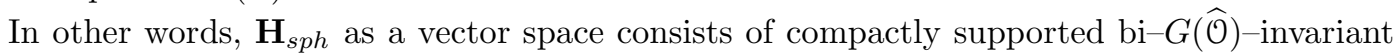
functions $G(\widehat{\mathcal{K}}) \rightarrow \overline{\mathbb{Q}}_{\ell}$ and the product is defined by

$$
f^{1} \star f^{2}(g)=\int_{G(\widehat{\mathcal{K}})} f^{1}(x) \cdot f^{2}\left(x^{-1} \cdot g\right) d x,
$$

where $d x$ is a Haar measure on $G(\widehat{\mathcal{K}})$ with $d x(G(\widehat{\mathcal{O}}))=1$.

The basic fact about $\mathbf{H}_{s p h}$ is that it is commutative. Moreover, one can show that when $G$ is split, $\mathbf{H}_{s p h}$ is isomorphic to the Grothendieck ring of the category of finite dimensional representations of the Langlands dual group $\check{G}$.

Now, let $\mathrm{I} \subset G(\widehat{\mathcal{O}})$ be the Iwahori subgroup, and let us consider the corresponding Hecke algebra, denoted $\mathbf{H}_{\mathrm{I}}$. For example, when $G$ is simply-connected, $\mathbf{H}_{\mathrm{I}}$ can be identified with the affine Hecke algebra attached to the root system of $G$.

Unlike $\mathbf{H}_{s p h}$, the algebra $\mathbf{H}_{\mathrm{I}}$ is non-commutative, and in this paper we will be concerned with its center, denoted $Z\left(\mathbf{H}_{\mathrm{I}}\right)$. The starting point is a theorem saying that $Z\left(\mathbf{H}_{\mathrm{I}}\right) \simeq \mathbf{H}_{s p h}$. Moreover, the map in one direction can be described very explicitly.

Let $\pi$ be a linear map from $\mathbf{H}_{\mathrm{I}}$ to the space of $G(\widehat{\mathcal{O}})-$ I-invariant functions defined by

$$
\pi(f)(g)=\int_{\mathrm{I} \backslash G(\widehat{\mathcal{O}})} f(x \cdot g) d x .
$$

It is easy to see that $\pi$ maps $Z\left(\mathbf{H}_{\mathrm{I}}\right)$ to $\mathbf{H}_{s p h}$ and a theorem of J. Bernstein (cf. [6], Theorem 2.13 or [13], Proposition 8.6) asserts that (at least when $G$ is split) this is an isomorphism. Our goal in this paper is to describe in some sense explicitly the inverse map to $\pi$. This will be done by realizing $\mathbf{H}_{s p h}$ and $\mathbf{H}_{\mathrm{I}}$ geometrically.

First, there exists a group-scheme (resp., an group-indscheme) over $\mathbb{F}_{q}$ whose set of $\mathbb{F}_{q^{-}}$ points identifies with $G(\widehat{\mathcal{O}})$ (resp., with $G(\widehat{\mathcal{K}})$ ). We will abuse the notation and denote these objects again by $G(\widehat{\mathcal{O}})$ and $G(\widehat{\mathcal{K}})$, respectively. In addition, there exists a subgroup $\mathrm{I} \subset G(\widehat{\mathcal{O}})$ of finite codimension, such that the quotient $G(\widehat{\mathcal{O}}) / \mathrm{I}$ is the flag variety $G / B$, where $B$ is a Borel subgroup of $G$. 
One can form the quotients $\mathrm{Gr}=G(\widehat{\mathcal{K}}) / G(\widehat{\mathcal{O}})$ and $\mathrm{Fl}=G(\widehat{\mathcal{K}}) / \mathrm{I}$, which will be indschemes over $\mathbb{F}_{q}$ and study the categories of perverse sheaves: $\mathrm{P}_{G(\widehat{\mathcal{O}})}(\mathrm{Gr})$ (resp., $\mathrm{P}_{\mathrm{I}}(\mathrm{Fl})$ ) will stand for the category of $G(\widehat{\mathcal{O}})$-equivariant perverse sheaves on $\mathrm{Gr}$ (resp., for I-equivariant perverse sheaves on $\mathrm{Fl}$ ).

The "faisceaux-fonctions" correspondence gives a map from the Grothendieck group of $\mathrm{P}_{G(\widehat{\mathcal{O}})}(\mathrm{Gr})$ to $\mathbf{H}_{s p h}$ and from the Grothendieck group of $\mathrm{P}_{\mathrm{I}}(\mathrm{Fl})$ to $\mathbf{H}_{\mathrm{I}}$. Moreover, one can introduce convolution functors $\mathrm{P}_{G(\widehat{\mathcal{O}})}(\mathrm{Gr}) \underset{\mathrm{Gr}}{\star} \mathrm{P}_{G(\widehat{\mathcal{O}})}(\mathrm{Gr}) \mapsto \mathrm{P}_{G(\widehat{\mathcal{O}})}(\mathrm{Gr})$ and $\mathrm{P}_{\mathrm{I}}(\mathrm{Fl}) \underset{\mathrm{Fl}}{\star} \mathrm{P}_{\mathrm{I}}(\mathrm{Fl}) \mapsto \mathrm{D}_{\mathrm{I}}^{b}(\mathrm{Fl})$ that will lift the $\star$ operations on $\mathbf{H}_{s p h}$ and $\mathbf{H}_{\mathrm{I}}$, respectively (cf. Sect. 1.1 for more details).

Now, we can formulate our task more precisely: we would like to construct a functor $Z$ : $\mathrm{P}_{G(\widehat{\mathcal{O}})}(\mathrm{Gr}) \rightarrow \mathrm{P}_{\mathrm{I}}(\mathrm{Fl})$, such that on the level of Grothendieck groups it induces the map $\pi^{-1}$.

It will turn out that this functor indeed exists and can be constructed using the operation of taking nearby cycles of a perverse sheaf. Namely, we will construct a 1-parametric family of schemes, which we will call $\mathrm{Fl}_{X}$, which degenerates the product $\mathrm{Gr} \times G / B$ to Fl. Then for $\mathcal{S} \in \mathrm{P}_{G(\widehat{\mathcal{O}})}(\mathrm{Gr}), Z(\mathcal{S})$ will be the nearby cycles of the product $\mathcal{S} \otimes \delta_{1_{G / B}}$.

Moreover, it will turn out that the functor $Z$ has some extremely favorable properties (cf. formulation of Theorem (1). In addition, since $Z$ is obtained by a nearby cycles construction, the perverse sheaves $Z(\mathcal{S})$ will possess an extra structure: that of a nilpotent endomorphism, coming from the monodromy. This phenomenon is invisible on the classical level (i.e. when one looks at the corresponding Grothendieck groups and not at the categories), and supposedly it carries a deep representation-theoretic meaning ([8]).

0.2. Conventions. This paper uses in an extensive way the language of indschemes and of perverse sheaves on them. Although the objects we will operate with are straightforward extensions of the corresponding finite-dimensional ones, not all of the definitions are present in the published literature, and for the reader's convenience we will review them in the Appendix, Sect. 6 .

As was mentioned before, $G$ is a connected reductive group over the base field $\mathbb{F}_{q}$. By $\operatorname{Rep}(G)$ we will denote the category of finite-dimensional $G$-representations. Throughout the paper, the notation $\mathcal{F}_{G}$ is reserved for principal $G$-bundles on various schemes and $\mathcal{F}_{G}^{0}$ we will denote the trivial $G$-bundle.

In several places in this paper we will use the concepts of a formal disc $\mathcal{D}$ and of a formal punctured disc $\mathcal{D}^{*}$. They will appear in the following circumstances:

Let $S=\operatorname{Spec}\left(\mathcal{O}_{S}\right)$ be an affine scheme. An $S$-family of $G$-bundles on $\mathcal{D}$ (resp., on $\mathcal{D}^{*}$ ) is by definition a tensor functor from $\operatorname{Rep}(G)$ to the tensor category of $S$-families of vector bundles on $\mathcal{D}$ (resp., $\left.\mathcal{D}^{*}\right)$, where the latter consists of finitely generated projective modules over $\mathcal{O}_{S}[[t]]$ (resp., $\left.\mathcal{O}_{S}((t))\right)$.

Let $\mathcal{D}_{k}=\operatorname{Spec}\left(\mathbb{F}_{q}[[t]] / t^{k+1}\right)$. It is easy to see that an $S$-family of $G$-bundles on $\mathcal{D}$ is the same as a compatible system of $G$-bundles on $\mathcal{D}_{k} \times S$. If $\mathcal{F}_{G}$ is an $S$-family on $\mathcal{D}$, we will denote by $\left.\mathcal{F}_{G}\right|_{\mathcal{D}^{*}}$ (resp., $\left.\mathcal{F}_{G}\right|_{\mathcal{D}_{k}}$ ) the corresponding induced family on $\mathcal{D}^{*}$ (resp., on $\mathcal{D}_{k}$ ).

An $S$-family of maps $\mathcal{D} \rightarrow G$ (resp., $\mathcal{D}^{*} \rightarrow G, \mathcal{D}_{k} \rightarrow G$ ) is is by definition a ring homomorphism $\mathcal{O}_{G} \rightarrow \mathcal{O}_{S}[[t]]$ (resp., $\left.\mathcal{O}_{G} \rightarrow \mathcal{O}_{S}((t)), \mathcal{O}_{G} \rightarrow \mathcal{O}_{S}[[t]] / t^{k+1}\right)$. The functor that attaches to $S$ the set of all $S$-families of maps $\mathcal{D} \rightarrow G$ (resp., $\mathcal{D}^{*} \rightarrow G, \mathcal{D}_{k} \rightarrow G$ ) is representable by a group-scheme (resp., by an group-indscheme, algebraic group) that will be denoted $G(\widehat{\mathcal{O}})$ (resp., $\left.G(\widehat{\mathcal{K}}), G(\widehat{\mathcal{O}})_{k}\right)$. We have: $G(\widehat{\mathcal{O}})=G(\widehat{\mathcal{O}})_{k}$. 
Let $y$ be a scheme, $H$ be an algebraic group and $y_{1}$ an $H$-torsor over $y$. Let, in addition, $y_{2}$ be an $H$-scheme. We will denote by $y_{1} \stackrel{H}{\times} y_{2}$ the associated fibration over $y$. If $\mathcal{T}$ is a perverse sheaf on $y$ and $\mathcal{S}$ is an $H$-equivariant perverse sheaf on $y_{2}$, we can form their twisted external product $\widetilde{\mathcal{}} \mathcal{S}$, which will be a perverse sheaf $y_{1} \stackrel{H}{\times} y_{2}$.

For a scheme $y, \overline{\mathbb{Q}}_{\ell} y$ will denote the constant sheaf on $y$ and for $y \in \mathcal{y}, \delta_{y}$ will denote the corresponding $\delta$-function sheaf.

Finally, we should mention that although we work over the ground field $\mathbb{F}_{q}$, all the results of this paper are valid over a ground field of characteristic zero.

0.3. Acknowledgments. This paper owes its existence to A. Beilinson: the very idea of obtaining "central" objects of $\mathrm{P}_{\mathrm{I}}(\mathrm{Fl})$ as nearby cycles of objects of $\mathrm{P}_{G(\widehat{\mathcal{O}})}(\mathrm{Gr})$ is an invention of his. 1 The author wishes to thank R. Bezrukavnikov for stimulating discussions. Finally, I am grateful to Ya. Varshavsky and the referee who pointed out numerous mistakes and whose comments helped me to improve the exposition.

\section{Formulation of the Results}

\subsection{Affine Grassmannian and affine flags.}

1.1.1. Consider the functor that associates to a scheme $S$ the set of pairs $\left(\mathcal{F}_{G}, \beta\right)$, where $\mathcal{F}_{G}$ is an $S$-family of $G$-bundles on $\mathcal{D}$ and $\beta$ is a trivialization of the corresponding family of $G-$ bundles on $\mathcal{D}^{*}$, i.e. $\beta:\left.\left.\mathcal{F}_{G}\right|_{\mathcal{D}^{*}} \rightarrow \mathcal{F}_{G}^{0}\right|_{\mathcal{D}^{*}}$. This functor is representable by an indscheme (cf. Sect. [), which we will denote by Gr, called the affine Grassmannian of $G$.

Here are the basic properties of Gr. First, Gr has a distinguished point $1_{\mathrm{Gr}} \in \mathrm{Gr}$ that corresponds to the pair $\left(\mathcal{F}_{G}^{0}, \beta^{0}\right)$, where $\beta^{0}$ is the tautological trivialization of the trivial bundle.

Consider the group-scheme $G(\widehat{\mathcal{O}})$ and the group-indscheme $G(\widehat{\mathcal{K}})$ (cf. Sect. 0.2). It is obvious that for a scheme $S, \operatorname{Hom}(S, G(\widehat{\mathcal{K}}))$ is the group of automorphisms of the trivial $S$-family of $G$-bundles on $\mathcal{D}^{*}$. Hence, $G(\widehat{\mathcal{K}})$ acts on $\mathrm{Gr}$ in a natural way, by changing the data of $\beta$.

It is known that the induced $G(\widehat{\mathcal{O}})$-action on Gr is "nice" (cf. Sect. 6 ). This means that Gr can be represented as a union of finite-dimensional closed subschemes, each of which is $G(\widehat{\mathcal{O}})$-stable. Therefore, we can introduce the category $\mathrm{P}_{G(\widehat{\mathcal{O}})}(\mathrm{Gr})$ of $G(\widehat{\mathcal{O}})$-equivariant perverse sheaves on $\mathrm{Gr}$ along with the corresponding derived category $\mathrm{D}_{G(\widehat{\mathcal{O}})}^{b}(\mathrm{Gr})$.

1.1.2. Now we will recall the convolution operation $\mathrm{P}_{G(\widehat{\mathcal{O}})}(\mathrm{Gr}) \times \mathrm{P}(\mathrm{Gr}) \mapsto \mathrm{D}^{b}(\mathrm{Gr})$.

For a non-negative integer $k$, let $\mathcal{G}_{k}$ be a $G(\widehat{\mathcal{O}})_{k}$-torsor over Gr, defined as the indscheme that represents the functor that associates to a scheme $S$ a triple $\left(\mathcal{F}_{G}, \beta, \gamma_{k}\right)$, where $\left(\mathcal{F}_{G}, \beta\right)$ are as above and $\gamma_{k}$ is a trivialization of $\left.\mathcal{F}_{G}\right|_{\mathcal{D}_{k}}$.

This defines a $G(\widehat{\mathcal{O}})$-torsor $\mathcal{G}$ over Gr, cf. Sect. 6. If we were to consider the total space of $\mathcal{G}$, it would be an indscheme (not of ind-finite type), isomorphic to $G(\widehat{\mathcal{K}})$. Therefore, one can loosely speak of Gr as being the quotient $G(\widehat{\mathcal{K}}) / G(\widehat{\mathcal{O}})$, which we will sometimes do in order to save notation.

The convolution diagram, denoted $\operatorname{Conv}_{\mathrm{Gr}}$, is the indscheme, associated to the $G(\widehat{\mathcal{O}})$-torsor $\mathcal{G}$ over $\mathrm{Gr}$ and the $G(\widehat{\mathcal{O}})$-scheme $\mathrm{Gr}$, i.e. $\mathrm{Conv}_{\mathrm{Gr}}=\mathcal{G} \stackrel{G(\widehat{\mathcal{O}})}{\times} \mathrm{Gr}$, according to our conventions. We again refer the reader to Sect. 6 for the explanation why Conv $_{\mathrm{Gr}}$ is a well-defined indscheme, as well as for the proof of the following lemma:

\footnotetext{
${ }^{1}$ As Beilinson points out, he was in turn inspired by T. Haines and R. Kottwitz, who proposed a similar idea in the framework of Shimura varieties, which has been realized in a recent preprint by T. Haines and B. C. Ngo.
} 
Lemma 1. The indscheme Conv $_{\mathrm{Gr}}$ represents the functor that attaches to a scheme $S$ a quadruple $\left(\mathcal{F}_{G}, \mathcal{F}_{G}^{1}, \widetilde{\beta}, \beta^{1}\right)$, where $\mathcal{F}_{G}$ and $\mathcal{F}_{G}^{1}$ are $S$-families of $G$-bundles on $\mathcal{D}, \widetilde{\beta}$ is an isomorphism $\left.\left.\mathcal{F}_{G}\right|_{\mathcal{D}^{*}} \rightarrow \mathcal{F}_{G}^{1}\right|_{\mathcal{D}^{*}}$ between the induced families of $G$-bundles on $\mathcal{D}^{*}$ and $\beta^{1}$ is an isomorphism $\left.\left.\mathcal{F}_{G}^{1}\right|_{\mathcal{D}^{*}} \rightarrow \mathcal{F}_{G}^{0}\right|_{\mathcal{D}^{*}}$.

There are two natural projections $p, p^{\prime}: \operatorname{Conv}_{\mathrm{Gr}} \rightarrow \mathrm{Gr}$. In the above functorial language, $p^{1}$ sends a quadruple $\left(\mathcal{F}_{G}, \mathcal{F}_{G}^{1}, \widetilde{\beta}, \beta^{1}\right)$ to $\left(\mathcal{F}_{G}^{1}, \beta^{1}\right)$ and $p\left(\mathcal{F}_{G}, \mathcal{F}_{G}^{1}, \widetilde{\beta}, \beta^{1}\right)=\left(\mathcal{F}_{G}, \beta^{1} \circ \widetilde{\beta}\right)$. Naively, one should picture the above projections as follows: if we identify $\operatorname{Conv}_{\mathrm{Gr}}$ with $G(\widehat{\mathcal{K}}) \stackrel{G(\widehat{\mathcal{O}})}{\times}$ $G(\widehat{\mathcal{K}}) / G(\widehat{\mathcal{O}})$, then $p^{1}$ is the projection on the first factor, i.e. $p^{1}\left(g_{1} \times g\right)=g_{1}$ and $p\left(g_{1} \times g\right)=g_{1} \cdot g$.

Thus, $p^{1}$ realizes $\operatorname{Conv}_{\mathrm{Gr}}$ as a fibration over $\mathrm{Gr}$, with the typical fiber isomorphic again to Gr. We are going to use the twisted external product construction, introduced in Sect. 0.2 and extended for ind-schemes in Sect. 6:

Starting with an object $\mathcal{T} \in \mathrm{P}(\mathrm{Gr})$ and an object $\mathcal{S} \in \mathrm{P}_{G(\widehat{\mathcal{O}})}(\mathrm{Gr})$, we can form their twisted external product $\mathcal{T} \widetilde{\otimes} \mathcal{S}$, which will be an object of $\mathrm{P}\left(\mathrm{Conv}_{\mathrm{Gr}}\right)$.

Finally we set $\mathcal{T}{ }_{\mathrm{Gr}} \mathcal{S}:=p_{!}(\mathcal{T} \widetilde{\otimes} \mathcal{S}) \in \mathrm{D}^{b}(\mathrm{Gr})$. It is easy to see that if $\mathcal{T}$ is also an object of $\mathrm{P}_{G(\widehat{\mathcal{O}})}(\mathrm{Gr})$, then $\mathcal{T}_{\mathrm{Gr}}^{\star \mathcal{S}}$ will belong to $\mathrm{D}_{G(\widehat{\mathcal{O}})}^{b}(\mathrm{Gr})$. Thus, $\underset{\mathrm{Gr}}{\star}$ induces a bi-functor from $\mathrm{P}_{G(\widehat{\mathcal{O}})}(\mathrm{Gr})$ to $\mathrm{D}_{G(\widehat{\mathcal{O}})}^{b}(\mathrm{Gr})$. On the level of Grothendieck groups, $\underset{\mathrm{Gr}}{\star}$ descends, of course, to the usual convolution product on the spherical Hecke algebra $\mathbf{H}_{s p h}$.

Remark. It follows from Lusztig's work 13 that for $\mathcal{S}_{1}, \mathcal{S}_{2} \in \mathrm{P}_{G(\widehat{\mathcal{O}})}(\mathrm{Gr})$, the convolution $\mathcal{S}_{1} \star \mathcal{S}_{\mathrm{Gr}}$ is again a perverse sheaf, i.e. the ${ }_{\mathrm{Gr}}^{\star}$-operation makes $\mathrm{P}_{G(\widehat{\mathcal{O}})}(\mathrm{Gr})$ into a monoidal category. Moreover, the fact that the spherical Hecke algebra $\mathbf{H}_{s p h}$ is commutative can be lifted to the categorical level: one can endow $\mathrm{P}_{G(\widehat{\mathcal{O}})}(\mathrm{Gr})$ with a commutativity constraint, i.e. $\mathrm{P}_{G(\widehat{\mathcal{O}})}(\mathrm{Gr})$ has a structure of a tensor category.

As a by-product of the results of this paper, we will construct the commutativity constraint $\mathcal{S}_{1} \underset{\mathrm{Gr}}{\star} \mathcal{S}_{2} \rightarrow \mathcal{S}_{2} \underset{\mathrm{Gr}}{\star} \mathcal{S}_{1}$ and, in addition, we will prove a strengthened version of Lusztig's theorem: we will show that for $\mathcal{S} \in \mathrm{P}_{G(\widehat{\mathcal{O}})}(\mathrm{Gr}), \mathcal{S} \widetilde{\otimes} \mathcal{T}$ is a perverse sheaf for any $\mathcal{T} \in \mathrm{P}(\mathrm{Gr})$.

1.1.3. Let us fix once and for all a Borel subgroup $B \subset G$, it corresponds to a distinguished point $1_{G / B}$ inside the flag variety $G / B$. The Iwahori group $\mathrm{I} \subset G(\widehat{\mathcal{O}})$ is by definition the preimage of $B$ under the natural projection $G(\widehat{\mathcal{O}}) \rightarrow G(\widehat{\mathcal{O}})_{0}=G$. We will denote by $\mathrm{I}_{k}$ the image of I under the projection $G(\widehat{\mathcal{O}}) \rightarrow G(\widehat{\mathcal{O}})_{k}$. We have: $\mathrm{I}=\mathrm{I}_{k}$.

The affine flag variety $\mathrm{Fl}$ is the indscheme associated to the $G$-bundle $\mathcal{G}_{0}$ over $\mathrm{Gr}$ and a $G$-variety $G / B$, i.e. $\mathrm{Fl}=\mathcal{G}_{0} \stackrel{G}{\times} G / B$. Thus, loosely speaking, $\mathrm{Fl}=G(\widehat{\mathcal{K}}) / \mathrm{I}$. We will denote by $\pi$ the natural projection $\mathrm{Fl} \rightarrow \mathrm{Gr}$.

Functorially, for a scheme $S$, the $\operatorname{set} \operatorname{Hom}(S, \mathrm{Fl})$ consists of triples $\left(\mathcal{F}_{G}, \beta, \epsilon\right)$, where $\mathcal{F}_{G}$ and $\beta$ are as in the definition of $\mathrm{Gr}$ and $\epsilon$ is a reduction of $\left.\mathcal{F}_{G}\right|_{\mathcal{D}_{0}}$ to $B$.

Let $1_{\mathrm{Fl}} \in \mathrm{Fl}$ be the distinguished point that corresponds to the triple $\left(\mathcal{F}_{G}^{0}, \beta^{0}, \epsilon^{0}\right)$, where $\left(\mathcal{F}_{G}^{0}, \beta^{0}\right)=1_{\mathrm{Gr}}$ and $\epsilon^{0}$ corresponds to the chosen Borel subgroup $B \subset G$.

The $G(\widehat{\mathcal{K}})$-action on Gr lifts in a natural way to an action on $\mathrm{Fl}$ and the induced actions of $G(\widehat{\mathcal{O}})$ and hence of I are "nice" in the sense of Sect. 6. Therefore, one may consider the categories $\mathrm{P}(\mathrm{Fl}), \mathrm{D}^{b}(\mathrm{Fl}), \mathrm{P}_{\mathrm{I}}(\mathrm{Fl})$ and $\mathrm{D}_{\mathrm{I}}(\mathrm{Fl})$.

As in the case of $\mathrm{Gr}$, one defines the ind-scheme Conv $v_{\mathrm{Fl}}$, which classifies the data of 6-tuples $\left(\mathcal{F}_{G}, \mathcal{F}_{G}^{1}, \widetilde{\beta}, \beta^{1}, \epsilon, \epsilon^{1}\right)$, where $\left(\mathcal{F}_{G}, \mathcal{F}_{G}^{1}, \widetilde{\beta}, \beta^{1}\right)$ are as in the definition of $\operatorname{Conv}_{G r}$ and $\epsilon$ (resp., $\left.\epsilon^{1}\right)$ is a reduction of $\left.\mathcal{F}_{G}\right|_{\mathcal{D}_{0}}$ (resp., of $\left.\mathcal{F}_{G}^{1}\right|_{\mathcal{D}_{0}}$ ) to $B$. 
Let $p^{1}$ and and $p$ denote the two projections from ConvFl to Fl. As in the previous case, we obtain a functor $\mathcal{T}, \mathcal{S} \mapsto \mathcal{T} \widetilde{\otimes} \mathcal{S}$ from $\mathrm{P}(\mathrm{Fl}) \times \mathrm{P}_{\mathrm{I}}(\mathrm{Fl})$ to $\mathrm{D}^{b}\left(\operatorname{Conv}_{\mathrm{Fl}}\right)$ and we set $\mathcal{T} \star \mathcal{F}:=p_{!}(\mathcal{T} \widetilde{\otimes} \mathcal{S})$.

However, Lusztig's theorem does not extend to the case of affine flags: the convolution functor $\underset{\mathrm{Fl}}{\star}$ does not preserve perversity, i.e. it maps $\mathrm{P}_{\mathrm{I}}(\mathrm{Fl}) \times \mathrm{P}_{\mathrm{I}}(\mathrm{Fl})$ to $\mathrm{D}_{\mathrm{I}}(\mathrm{Fl})$. In addition, there certainly is no isomorphism $\mathcal{S}_{1} \underset{\mathrm{Fl}}{\star} \mathcal{S}_{2} \rightarrow \mathcal{S}_{2} \underset{\mathrm{Fl}}{\star} \mathcal{S}_{1}$ since the corresponding equality is not true even on the Grothendieck group level (the Iwahori Hecke algebra $\mathbf{H}_{\mathrm{I}}$ is not commutative).

\subsection{The functor $Z$.}

1.2.1. The main result of this paper is the following theorem:

Theorem 1. There exists a functor: $Z: \mathrm{P}_{G(\widehat{\mathcal{O}})}(\mathrm{Gr}) \rightarrow \mathrm{P}_{\mathrm{I}}(\mathrm{Fl})$ possessing the following properties:

(a) For $\mathcal{S} \in \mathrm{P}_{G(\widehat{\mathcal{O}})}(\mathrm{Gr})$ and an arbitrary perverse sheaf $\mathcal{T}$ on $\mathrm{Fl}$, the convolution $\mathcal{T}_{\mathrm{Fl}}^{\star Z(\mathcal{S})}$ is a perverse sheaf.

(b) For $\mathcal{S} \in \mathrm{P}_{G(\widehat{\mathcal{O}})}(\mathrm{Gr})$ and $\mathcal{T} \in \mathrm{P}_{\mathrm{I}}(\mathrm{Fl})$ there is a canonical isomorphism $Z(\mathcal{S}) \underset{\mathrm{Fl}}{\star} \mathcal{T} \simeq \mathcal{T} \star Z(\mathcal{F l})$.

(c) We have $Z\left(\delta_{1_{\mathrm{Gr}}}\right)=\delta_{1_{\mathrm{Fl}}}$ and for $\mathcal{S}^{1}, \mathcal{S}^{2} \in \mathrm{P}_{G(\widehat{\mathcal{O}})}(\mathrm{Gr})$ there is a canonical isomorphism $Z\left(\mathcal{S}^{1}\right) \underset{\mathrm{Fl}}{\star} Z\left(\mathcal{S}^{2}\right) \simeq Z\left(\mathcal{S}_{\mathrm{Gr}}^{1} \underset{\mathcal{S}^{2}}{ }\right)$.

(d) For $\mathcal{S} \in \mathrm{P}_{G(\widehat{\mathcal{O}})}(\mathrm{Gr})$, we have $\pi_{!}(Z(\mathcal{S})) \simeq \mathcal{S}$.

Property (d) above insures that the composition

$$
K\left(\mathrm{P}_{G(\widehat{\mathcal{O}})}(\mathrm{Gr})\right) \stackrel{Z}{\rightarrow} K\left(\mathrm{P}_{\mathrm{I}}(\mathrm{Fl})\right) \rightarrow \mathbf{H}_{\mathrm{I}}
$$

equals $K\left(\mathrm{P}_{G(\widehat{\mathcal{O}})}(\mathrm{Gr})\right) \rightarrow \mathbf{H}_{s p h} \simeq Z\left(\mathbf{H}_{\mathrm{I}}\right)$, i.e. Theorem 1 fulfills our promise to construct geometrically the inverse of the map $Z\left(\mathbf{H}_{\mathrm{I}}\right) \rightarrow \mathbf{H}_{s p h}$.

1.2.2. An additional basic structure of the functor $Z$ is described by the following theorem:

Theorem 2. The functor $Z$ carries a nilpotent (monodromy) endomorphism $M$

$$
M_{\mathcal{S}}: Z(\mathcal{S}) \rightarrow Z(\mathcal{S})(-1), \text { for } \mathcal{S} \in \mathrm{P}_{G(\widehat{\mathcal{O}})}(\mathrm{Gr}),
$$

which is compatible with the isomorphisms of Theorem $\mathbb{Q}(c)$ : For $\mathcal{S}_{1}, \mathcal{S}_{2} \in \mathrm{P}_{G(\widehat{\mathcal{O}})}(\mathrm{Gr})$ the square

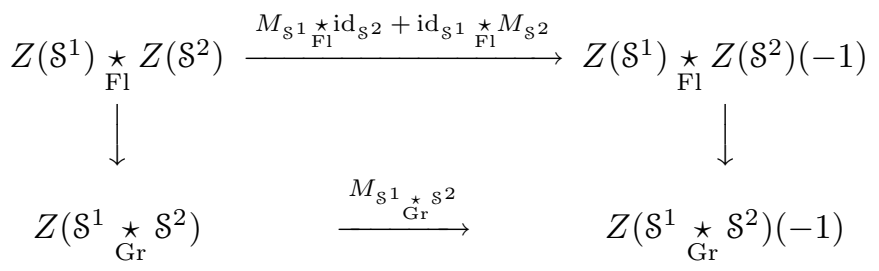

commutes.

1.2.3. Let explain the first non-trivial example of how Theorem 1 works for $G=G L(2)$. Consider the following closed $G(\widehat{\mathcal{O}})$-stable subscheme $Y_{0}$ of $\mathrm{Gr}$ :

By definition, for $G=G L(2)$, Gr classifies lattices in $\widehat{\mathcal{K}} \oplus \widehat{\mathcal{K}}$ (i.e. $\widehat{\mathcal{O}}$-submodules $\mathcal{L} \subset \widehat{\mathcal{K}} \oplus \widehat{\mathcal{K}}$ of rank 2) and $Y_{0}$ corresponds to those $\mathcal{L}$ which are contained in $\mathcal{L}^{0}:=\widehat{\mathcal{O}} \oplus \widehat{\mathcal{O}}$ with $\operatorname{dim}\left(\mathcal{L}^{0} / \mathcal{L}\right)=1$.

By construction, $Y_{0}$ is isomorphic to the projective line $\mathbb{P}^{1}$. We take $\mathcal{S} \in \mathrm{P}_{G(\widehat{\mathcal{O}})}(\mathrm{Gr})$ to be $\overline{\mathbb{Q}}_{\ell Y_{0}}[1]$. In this case, the construction which will be discussed in the next section reduces to the 
usual Picard-Lefschetz situation and the perverse sheaf $Z(\mathcal{S}) \in \mathrm{P}_{\mathrm{I}}(\mathrm{Fl})$ can be described very explicitly.

First, $Z(\mathcal{S})$ will be supported on $\pi^{-1}\left(Y_{0}\right) \subset \mathrm{Fl}$. Now let $Y_{1}$ and $Y_{2}$ be the following two subschemes of $\pi^{-1}\left(Y_{0}\right)$ :

By definition, $\mathrm{Fl}$ classifies lattices $\mathcal{L}$ as above plus a choice of a line $\ell \subset \mathcal{L} / t \cdot \mathcal{L}$, where $t$ is the uniformizer of $\widehat{\mathcal{O}}$. The chosen Borel subgroup $B \subset G L(2)$ fixes a line $\ell^{0} \subset \mathcal{L}^{0} / t \cdot \mathcal{L}^{0} \simeq \mathbb{F}_{q} \oplus \mathbb{F}_{q}$ and $Y_{1}$ corresponds to the lattice $L^{1}=\operatorname{ker}\left(\mathcal{L}_{0} \rightarrow \mathbb{F}_{q} \oplus \mathbb{F}_{q} \rightarrow \mathbb{F}_{q} \oplus \mathbb{F}_{q} / \ell^{0}\right)$ and an arbitrary $\ell$. On the contrary, $Y_{2}$ corresponds to an arbitrary $\mathcal{L} \in Y_{0}$, but $\ell$ must be the kernel of the map $\mathcal{L} / t \cdot \mathcal{L} \rightarrow \mathcal{L}^{0} / t \cdot \mathcal{L}^{0}$. Both $Y_{1}$ and $Y_{2}$ are isomorphic to $\mathbb{P}^{1}$ and their intersection in $\mathrm{Fl}$ is a point-scheme, which we will denote by $Y_{3}$.

We claim that $Z(\mathcal{S})$ has the following form: it has a three-step filtration

$$
0=F_{0} \subset F_{1} \subset F_{2} \subset F_{3}=Z(\mathcal{S}),
$$

such that $F_{1} \simeq \delta_{Y_{3}}, F_{2} / F_{1} \simeq \overline{\mathbb{Q}}_{\ell Y_{1}}[1] \oplus \overline{\mathbb{Q}}_{\ell Y_{2}}[1]$ and $F_{3} / F_{2} \simeq \delta_{Y_{3}}(-1)$. Moreover, the monodromy map $M_{\mathcal{S}}$ acts as follows:

$$
Z(\mathcal{S}) \rightarrow F_{3} / F_{2} \simeq \delta_{Y_{3}}(-1) \simeq F_{1}(-1) \hookrightarrow Z(\mathcal{S})(-1) .
$$

\section{Construction-I}

\subsection{Global version of the affine Grassmannian.}

2.1.1. To carry out our constructions we will need to choose a curve $X$, which is smooth, but not necessarily complete, and an $\mathbb{F}_{q}$-point point $x \in X$. In what follows, we will choose once and for all an identification of the completed local ring $\widehat{\mathcal{O}}_{x}$ with $\widehat{\mathcal{O}}$.

The starting point is the following result of [5]:

Lemma 2. The indscheme Gr represents the following functor: $\operatorname{Hom}(S, \mathrm{Gr})$ is the set of pairs $\left(\mathcal{F}_{G}, \beta\right)$, where $\mathcal{F}_{G}$ is a $G$-bundle on $X \times S$ and $\beta$ is its trivialization over $(X \backslash x) \times S$.

Remark. Of course, if one has a pair $\left(\mathcal{F}_{G}, \beta\right)$ as in the proposition, one can restrict it to the formal disc around $x$ and thus obtain a point of $\mathrm{Gr}$ in the original definition. The meaning of Lemma 2 is that this restriction is a bijection between the data on $X$ and that on the formal disc.

2.1.2. Let Aut denote the pro-algebraic group of automorphisms of $\widehat{\mathcal{O}}$, i.e. for an affine scheme $S, \operatorname{Hom}(S$, Aut $)$ consists of all $\mathcal{O}_{S}$-linear continuous automorphisms of $\mathcal{O}_{S}[[t]]$. By definition, Aut is the projective limit of the groups Aut ${ }_{k}$, where Aut $k$ is the group of automorphisms of $\mathbb{F}_{q}[[t]] / t^{k+1}$. This group acts in a canonical way on $G(\widehat{\mathcal{O}}), G(\widehat{\mathcal{K}})$ and on Gr; moreover, its action on Gr is "nice" in the sense of Sect. 6.

In addition, there is a canonical Aut-torsor $\mathcal{X}$ over $X$ : for an affine scheme $S$, an $S$-point of $X$ is a pair $y: S \rightarrow X$ and a continuous $\mathcal{O}_{S}$-linear isomorphism between $\mathcal{O}_{S}[[t]]$ and the completion of $\mathcal{O}_{X \times S}$ along the graph $\Gamma_{y}$ of the map $y$.

We define the global version of $\operatorname{Gr}$ over $X$, denoted $\operatorname{Gr}_{X}$, as an indscheme associated to the Aut-torsor $X$ over $X$ and the Aut-scheme Gr, i.e. $\operatorname{Gr}_{X}:=X \stackrel{\text { Aut }}{\times}$ Gr, cf. Sect. 6 .

By invoking again the theorem of [5], we obtain the following:

Lemma 3. The indscheme $\operatorname{Gr}_{X}$ represents the following functor: $\operatorname{Hom}\left(S, \mathrm{Gr}_{X}\right)$ is the set of triples $\left(y, \mathcal{F}_{G}, \beta\right)$, where $y$ is an $S$-point of $X, \mathcal{F}_{G}$ is a $G$-bundle on $X \times S$ and $\beta$ is a trivialization $\left.\left.\mathcal{F}_{G}\right|_{X \times S \backslash \Gamma_{y}} \rightarrow \mathcal{F}_{G}^{0}\right|_{X \times S \backslash \Gamma_{y}}$, where $\Gamma_{y} \subset X \times S$ is the graph of $y: S \rightarrow X$.

We will denote by $\operatorname{Gr}_{X \backslash x}$ (resp., $\mathrm{Gr}_{x}$ ) the preimage of $X \backslash x$ (resp., of $x \in X$ ) under the natural projection $\mathrm{Gr}_{X} \rightarrow X$. 
2.1.3. An important observation is that to an object $\mathcal{S} \in \mathrm{P}_{G(\widehat{\mathcal{O}})}(\mathrm{Gr})$, one can attach in a canonical way a perverse sheaf on $\mathrm{Gr}_{X}$. First, we have the following assertion:

Proposition 1. Every $G(\widehat{\mathcal{O}})$-equivariant perverse sheaf on $\mathrm{Gr}$ is automatically equivariant with respect to Aut.

Proof. To prove the proposition, it is enough to show that over an algebraic closure $\overline{\mathbb{F}}_{q}$ of $\mathbb{F}_{q}$, the category $\mathrm{P}_{G(\widehat{\mathcal{O}})}(\mathrm{Gr})$ is semi-simple and every irreducible object in it is Aut-equivariant. Indeed, this would imply that every object of $\mathrm{P}_{G(\widehat{\mathcal{O}})}(\mathrm{Gr})$ has a form $\underset{i}{\oplus} \mathcal{S}_{i} \otimes V_{i}$, where $\mathcal{S}_{i}$ is absolutely irreducible (and hence Aut-equivariant) and $V_{i}$ is a "perverse sheaf over $\operatorname{Spec}\left(\mathbb{F}_{q}\right)$ ", i.e. a vector space acted on by the Frobenius.

The two facts mentioned above are well-known and we include the proof for completeness. First of all, every irreducible $G(\widehat{\mathcal{O}})$-equivariant perverse sheaf on $\mathrm{Gr}$ is an intersection cohomology sheaf on a closure of a $G(\widehat{\mathcal{O}})$-orbit and each such orbit is Aut-stable.

To prove the semi-simplicity assertion, we must show that if $Y^{\prime}$ and $Y$ are two $G(\widehat{\mathcal{O}})$-orbits, then $\operatorname{Ext}^{1}\left(\mathrm{IC}_{\bar{Y}}, \mathrm{IC}_{\overline{Y^{\prime}}}\right)=0$.

First, let us assume that $Y=Y^{\prime}$. The natural map

$$
\operatorname{Ext}^{1}\left(\operatorname{IC}_{\bar{Y}}, \operatorname{IC}_{\overline{Y^{\prime}}}\right) \rightarrow \operatorname{Ext}^{1}\left(\overline{\mathbb{Q}}_{\ell Y}, \overline{\mathbb{Q}}_{\ell Y}\right)
$$

is an injection. However, the right-hand side is nothing but $H^{1}\left(Y, \overline{\mathbb{Q}}_{\ell}\right)$, and it vanishes, since any $G(\widehat{\mathcal{O}})$-orbit $Y$ is a isomorphic to a principal bundle over a (partial) flag variety of the group $G$ with a unipotent structure group.

Thus, let $Y \neq Y^{\prime}$ and without restricting the generality, we can assume that $Y^{\prime} \subset \bar{Y}$. (It is easy to see that when neither $Y^{\prime} \subset \bar{Y}$ nor $Y \subset \overline{Y^{\prime}}$, the above $\operatorname{Ext}^{1}$ is automaticaly zero). It is enough to show that the $*$-restriction of $\mathrm{IC}_{\bar{Y}}$ to $Y^{\prime}$ lives in the perverse cohomological degrees $\leq-2$. It is known due to [12], that the stalks of $\operatorname{IC}_{\bar{Y}}$ have the parity vanishing property. If Since the dimensions of $Y$ and $Y^{\prime}$ have the same parity, $\left.\operatorname{IC}_{\bar{Y}}\right|_{Y^{\prime}}$ has perverse cohomologies only in the even degrees. In particular, its -1-st perverse cohomology sheaf is 0 , which is what we had to prove. 3

Thus, starting with $\mathcal{S} \in \mathrm{P}_{G(\widehat{\mathcal{O}})}(\mathrm{Gr})$, or, more generally, with $\mathcal{S} \in \mathrm{P}_{\text {Aut }}(\mathrm{Gr})$, we can attach to it a perverse sheaf $\delta_{X} \in \mathrm{P}\left(\mathrm{Gr}_{X}\right)$, by taking the twisted external product with the constant perverse sheaf $\overline{\mathbb{Q}}_{\ell}[1]$ on $X$. We will denote the restriction of $\mathcal{S}_{X}$ to $\operatorname{Gr}_{X \backslash x}$ by $\mathcal{S}_{X \backslash x}$.

\subsubsection{The following will be useful in the sequel:}

Starting from the Aut-torsor $X$ over $X$ and the group-scheme $G(\widehat{\mathcal{O}})$, we can form a groupscheme $G(\widehat{\mathcal{O}})_{X}$ over $X$ by setting $G(\widehat{\mathcal{O}})_{X}:=X \stackrel{\text { Aut }}{\times} G(\widehat{\mathcal{O}})$. By construction, $G(\widehat{\mathcal{O}})_{X}$ acts on $\operatorname{Gr}_{X}$ and for $\mathcal{S} \in \mathrm{P}_{G(\widehat{\mathcal{O}})}(\mathrm{Gr})$ the perverse sheaf $\mathcal{S}_{X}$ are equivariant with respect to this action.

Proposition 2. Let $S$ be a scheme, let $\left(y, \mathcal{F}_{G}, \beta\right)$ be an $S$-point of $\operatorname{Gr}_{X}$ and let $\phi: X \times S \rightarrow G$ be a map. Consider another $S-$ point of $\mathrm{Gr}_{X}$ equal to $\left(y, \mathcal{F}_{G}, \phi \circ \beta\right)$. Then, for $\mathcal{S} \in \mathrm{P}_{G(\widehat{\mathcal{O}})}(\mathrm{Gr})$ the pull-backs of $\mathcal{S}_{X}$ under these two maps from $\operatorname{Gr}_{X}$ to $S$ are canonically isomorphic

\footnotetext{
${ }^{2}$ Following the referee's suggestion, since the last assertion was not stated explicitly for the affine case in 12 , we will give a self-conated proof at the end of Sect. 6 .

${ }^{3}$ The above proof that $\operatorname{Ext}^{1}\left(\mathrm{IC}_{\bar{Y}}, \mathrm{IC}_{\overline{Y^{\prime}}}\right)=0$ over an algebraically closed field relies on the Kazhdan-Lusztig parity vanishing assertion. However, in the recent paper 11 it was shown that the fact that $\operatorname{Ext}^{1}\left(\mathrm{IC}_{\bar{Y}}, \mathrm{IC}_{\overline{Y^{\prime}}}\right)=0$ for $Y=Y^{\prime}$ formally implies the vanishing in the general case.
} 
Proof. By taking Taylor expansions of the map $\phi$, we obtain a map $\widehat{\phi}: X \times S \rightarrow G(\widehat{\mathcal{O}})_{X}$. Consider the map $S \rightarrow G(\widehat{\mathcal{O}})_{X} \underset{X}{\times} \operatorname{Gr}_{X}$ obtained from the first map $S \rightarrow \operatorname{Gr}_{X}$ and

$$
S \stackrel{y \times \text { id }}{\longrightarrow} X \times S \stackrel{\widehat{\phi}}{\rightarrow} G(\widehat{\mathcal{O}})_{X}
$$

Our two $S$-points of $\mathrm{Gr}_{X}$ are the compositions of the above map followed by the projection $G(\widehat{\mathcal{O}})_{X} \times \operatorname{Gr}_{X} \rightarrow \operatorname{Gr}_{X}$ in the first case, and by the action map $G(\widehat{\mathcal{O}})_{X} \times \operatorname{Gr}_{X} \rightarrow \operatorname{Gr}_{X}$ in the second one.

Hence, the proposition follows from the $G(\widehat{\mathcal{O}})_{X}$-equivariance of $\mathcal{S}_{X}$.

\subsection{The nearby cycles construction.}

2.2.1. First, we will construct an indscheme $\mathrm{Fl}_{X}$ over $X$. We define $\operatorname{Hom}\left(S, \mathrm{Fl}_{X}\right)$ to be the set of quadruples $\left(y, \mathcal{F}_{G}, \beta, \epsilon\right)$, where $\left(y, \mathcal{F}_{G}, \beta\right)$ are as in Lemma 3 and $\epsilon$ is a data of a reduction of $\left.\mathcal{F}_{G}\right|_{x \times S}$ to $B$.

Obviously, $\mathrm{Fl}_{X}$ is a fibration over $\mathrm{Gr}_{X}$ with the typical fiber $G / B$. We will denote the projection $\mathrm{Fl}_{X} \rightarrow \mathrm{Gr}_{X}$ by $\pi_{X}$. Let $\mathrm{Fl}_{X \backslash x}$ and $\mathrm{Fl}_{x}$ be the corresponding subschemes of $\mathrm{Fl}_{X}$.

Proposition 3. We have canonical isomorphisms $\mathrm{Fl}_{X \backslash x} \simeq \mathrm{Gr}_{X \backslash x} \times G / B$ and $\mathrm{Fl}_{x} \simeq \mathrm{Fl}$.

Proof. Let $\left(y, \mathcal{F}_{G}, \beta, \epsilon\right)$ be an $S$-point of $\mathrm{Fl}_{X}$ with $\Gamma_{y} \cap(x \times S)=\emptyset$. Then the data of $\beta$ trivializes $\left.\mathcal{F}_{G}\right|_{x \times S}$. Therefore, $\epsilon$ is a reduction to $B$ of the trivial $G$-bundle on $S$, i.e. a map $S \rightarrow G / B$

This defines a map $\mathrm{Fl}_{X \backslash x} \simeq \mathrm{Gr}_{X \backslash x} \times G / B$ and it is straightforward to see that it is an isomorphism.

The fact that $\mathrm{Fl}_{x} \simeq \mathrm{Fl}$ follows immediately from Lemma 2.

2.2.2. Let us recall the general formalism of the nearby cycles functor. Let $y$ be a scheme mapping to $X$ and let $y_{X \backslash x}$ and $y_{x}$ be its corresponding subschemes.

We have a functor

$$
\Psi_{y}: \mathrm{D}^{b}\left(y_{X \backslash x}\right) \rightarrow \mathrm{D}^{b}\left(y_{x}\right)
$$

whose basic property is that it maps $\mathrm{P}\left(y_{X \backslash x}\right)$ to $\mathrm{P}\left(y_{x}\right)$, according to [1].

2.2.3. We apply $\Psi$ for $y=\mathrm{Fl}_{X}$. Using Sect. 2.1.3 and Lemma 3, we can construct a functor from $\mathrm{P}_{\text {Aut }}(\mathrm{Gr})$ to $\mathrm{P}\left(\mathrm{Fl}_{X \backslash x}\right)$ :

$$
\mathcal{S} \mapsto \mathcal{S}_{X \backslash x} \otimes \delta_{1_{G / B}} .
$$

We set $Z(\mathcal{S})=\Psi_{\mathrm{Fl}_{X}}\left(\mathcal{S}_{X \backslash x} \otimes \delta_{1_{G / B}}\right) \in \mathrm{P}(\mathrm{Fl})$.

It is straightforward to see that $Z\left(\delta_{1_{\mathrm{Gr}}}\right) \simeq \delta_{1_{\mathrm{Fl}}}$. Indeed, we have a canonical section $1_{\mathrm{Fl}_{X}}$ : $X \rightarrow \mathrm{Fl}_{X}$ that sends $y$ the quadruple $\left(y, \mathcal{F}_{G}^{0}, \beta^{0}, \epsilon^{0}\right)$ and $1_{\mathrm{Fl}_{X \backslash x}}=1_{\mathrm{Gr}_{X \backslash x}} \times 1_{G / B}, 1_{\mathrm{Fl}_{x}}=1_{\mathrm{Fl}}$.

\subsection{I-equivariance.}

${ }^{4}$ A priori, for $\mathcal{S} \in \mathrm{D}^{b}\left(y_{X \backslash x}\right), \Psi_{y}(\mathcal{S})$ is defined only over $\overline{\mathbb{F}}_{q}$. To endow it with an $\mathbb{F}_{q}$-structure one needs to choose a splitting from the Galois group $\operatorname{Gal}\left(\overline{\mathbb{F}}_{q} / \mathbb{F}_{q}\right)$ to the Galois group of the field of fractions of the henselization of the local ring $\mathcal{O}_{x}$. From now on we choose such a splitting. 
2.3.1. By construction, $\mathcal{S} \rightarrow Z(\mathcal{S})$ is a functor between $\mathrm{P}_{\text {Aut }}(\mathrm{Gr})$ and $\mathrm{P}(\mathrm{Fl})$. The next proposition asserts that it defines a functor $\mathrm{P}_{G(\widehat{\mathcal{O}})}(\mathrm{Gr}) \rightarrow \mathrm{P}_{\mathrm{I}}(\mathrm{Fl})$, as required in Theorem 1 .

Proposition 4. For $\mathcal{S} \in \mathrm{P}_{G(\widehat{\mathcal{O}})}(\mathrm{Gr})$, the perverse sheaf $Z(\mathcal{S})$ on $\mathrm{Fl}$ is $\mathrm{I}$-equivariant.

Proof. Let $Y$ be the support of $Z(\mathcal{S})$ in Fl. Choose an integer $k$ so that I acts on $Y$ through the quotient $\mathrm{I} \rightarrow \mathrm{I}_{k}$. Thus, we have to show that $Z(\mathcal{S})$ is $\mathrm{I}_{k}$-equivariant.

Let $\mathrm{I}_{g l o b}$ be the sheaf of groups (on the category of all schemes with Zarisky topology), which attaches to a scheme $S$ the group of maps from the localization of $X \times S$ around $x \times S$ to $G$, with the condition that $x \times S$ maps to $B$. By taking Taylor expansions at $x$, we obtain a map of sheaves $\mathrm{I}_{g l o b} \rightarrow \underline{\mathrm{I}}$ (the underline means "the sheaf represented by").

Lemma 4. The composition $\mathrm{I}_{g l o b} \rightarrow \underline{\mathrm{I}} \rightarrow \underline{\mathrm{I}_{k}}$ is a surjection of sheaves of groups.

The proof will be given below. From this lemma we infer that it suffices to prove the following:

Let $S$ be a smooth scheme and let $U$ be an open subset in $X \times S$ containing $x \times S$. Let $\phi: U \rightarrow G$ be a section of $\mathrm{I}_{g l o b}$. We have two maps from $S \times \mathrm{Fl} \rightarrow \mathrm{Fl}$ : one is the projection on the second factor and the other one is obtained by composing $S \rightarrow \mathrm{I}_{g l o b} \rightarrow \underline{\mathrm{I}}$ and the action of I on Fl. We must show that the pull-backs of $Z(\mathcal{S})$ under these two maps from Fl to $S \times \mathrm{Fl}$ are isomorphic.

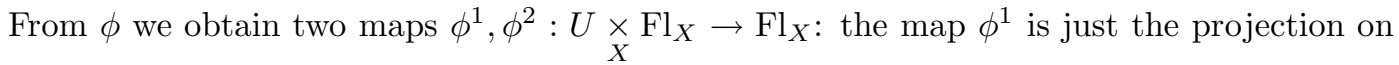
the second factor and the map $\phi^{2}$ is described as follows:

Let $S^{\prime}$ be another scheme and let us consider an $S^{\prime}$-point of $\underset{X}{U} \underset{\mathrm{Fl}}{X}$. This amounts to having an $S^{\prime}$-point $\left(y, \mathcal{F}_{G}, \beta, \epsilon\right)$ of $\mathrm{Fl}_{X}$ and a map $g: S^{\prime} \rightarrow S$ such that the map $S^{\prime} \stackrel{y \times g}{\longrightarrow} X \times S$ factors through $U \subset X \times S$.

Let $U^{\prime}$ denote the preimage of $U$ under the map $X \times S^{\prime} \stackrel{\text { id } \times g}{\longrightarrow} X \times S$. Using $\phi$, we obtain a map $\phi^{\prime}: U^{\prime} \rightarrow G$. We need to produce another $S^{\prime}$-point $\left(y, \mathcal{F}_{G}^{\phi}, \beta^{\phi}, \epsilon^{\phi}\right)$ of $\mathrm{Fl}_{X}$.

By definition, $\mathcal{F}_{G}^{\phi}$ is the trivial bundle on $X \times S^{\prime} \backslash \Gamma_{y}$ and is identified with $\mathcal{F}_{G}$ over $U^{\prime}$. We have: $U^{\prime} \cup\left(X \times S \backslash \Gamma_{y}\right)=X \times S^{\prime}$. Therefore, to define $\mathcal{F}_{G}^{\phi}$ we need to define a gluing isomorphism $\left.\left.\mathcal{F}_{G}^{0}\right|_{U^{\prime} \cap\left(X \times S^{\prime} \backslash \Gamma_{y}\right)} \simeq \mathcal{F}_{G}\right|_{U^{\prime} \cap\left(X \times S^{\prime} \backslash \Gamma_{y}\right)}$. The latter is obtained by composing $\beta$ and $\phi^{\prime}$. The data of $\epsilon^{\phi}$ is by definition induced by $\epsilon$, via the identification $\left.\left.\mathcal{F}_{G}^{\phi}\right|_{U^{\prime}} \simeq \mathcal{F}_{G}\right|_{U^{\prime}}$, since $U^{\prime}$ contains $x \times S^{\prime}$. The data of $\beta^{\phi}$ follows from the construction.

Both $\phi^{1}$ and $\phi^{2}$ are maps of schemes over $X$. Note that their values over $x$ are the two maps $S \times \mathrm{Fl} \rightarrow \mathrm{Fl}$ described above. In addition, $\phi$ defines a map $\widehat{\phi}: U \rightarrow G(\widehat{\mathcal{O}})_{X}$, and over $X \backslash x$ the maps $\phi^{1}$ and $\phi^{2}$ factor by means of $\widehat{\phi}$ through the action of $G(\widehat{\mathcal{O}})_{X \backslash x}$ on $\mathrm{Fl}_{X \backslash x} \simeq \operatorname{Gr}_{X \backslash x} \times G / B$, as in Proposition 2.

Both $\phi^{1}$ and $\phi^{2}$ are smooth and it is well-known that the functor of nearby cycles commutes with pull-backs under smooth morphisms. Therefore, it suffices to show that

$$
\Psi_{U \times \mathrm{Fl}_{X}}\left(\phi^{1 *}\left(\mathcal{S}_{X \backslash x} \otimes \delta_{1_{G / B}}\right)\right) \simeq \Psi_{U \times \mathrm{Fl}_{X}}\left(\phi^{2 *}\left(\mathcal{S}_{X \backslash x} \otimes \delta_{1_{G / B}}\right)\right) .
$$

However, as in Proposition 20 we obtain that $\phi^{1 *}\left(\mathcal{S}_{X \backslash x} \otimes \delta_{1_{G / B}}\right) \simeq \phi^{2 *}\left(\mathcal{S}_{X \backslash x} \otimes \delta_{1_{G / B}}\right)$.

\subsubsection{Finally, let us prove Lemma 4 .}

Proof. We will prove that if $S$ is affine, the map $\mathrm{I}_{g l o b}(S) \rightarrow \operatorname{Hom}\left(S, \mathrm{I}_{k}\right)$ is a surjection. 
The proof goes by induction. The assertion is obvious for $k=0$, since $I_{0}=B$. Therefore, it suffices to show that any map $g_{k}: \mathcal{D}_{k} \times S \rightarrow G$ whose restriction to $\mathcal{D}_{k-1} \times S$ is trivial, can be lifted to a map from the localization $(X \times S)_{x \times S}$ to $G$.

However, the group of maps $\left\{g_{k}\right\}$ as above is abelian and is isomorphic to the group of functions on $S$ with values in the Lie algebra of $G$. In addition, we know that $\operatorname{Lie}(G)$ is spanned by 1-dimensional subspaces that correspond to subgroups of $G$ isomorphic to either $\mathbb{G}_{m}$ or $\mathbb{G}_{a}$. Hence, it suffices to analyze separately these two cases.

For $\mathbb{G}_{a}$ the assertion is obvious: we can assume that $X$ is affine and then any function on $\mathcal{D}_{k} \times S$ can be extended to a function on $X \times S$. Similarly for $\mathbb{G}_{m}$ :

Let $t$ be a coordinate on $X$ around $x$. Our map $g_{k}$ is a $k$-jet of a regular function on $X \times S$ of the form $1+t^{k} \cdot f$, where $f$ is a function on $S$. This $1+t^{k} \cdot f$ is the required map $(X \times S)_{x \times S} \rightarrow \mathbb{G}_{m}$.

Thus, we have constructed the functor $Z$. However, in order to prove Theorem 1, we will need to interpret the convolutions $Z(\mathcal{S}) \underset{\text { Fl }}{\star} \mathcal{T}$ and $\underset{\text { Fl }}{\star} Z(\mathcal{S})$ too, in terms of nearby cycles.

\section{Construction-II}

\subsection{The indscheme $\mathrm{Fl}_{X}^{\prime}$.}

3.1.1. Our main tool will be the indscheme $\mathrm{Fl}_{X}^{\prime}$ over $X$ defined as follows:

For a scheme $S, \operatorname{Hom}\left(S, \mathrm{Fl}_{X}^{\prime}\right)$ is the set of quadruples $\left(y, \mathcal{F}_{G}, \beta^{\prime}, \epsilon\right)$, where $\left(y, \mathcal{F}_{G}, \epsilon\right)$ are as in the definition of $\mathrm{Fl}_{X}$, but $\beta^{\prime}$ is now a trivialization of $\mathcal{F}_{G}$ off the divisor $\Gamma_{y} \cup(x \times S)$.

Analogously, we introduce the indscheme $\operatorname{Gr}_{X}^{\prime}$ : $\operatorname{Hom}\left(S, \mathrm{Gr}_{X}^{\prime}\right)$ is the set of triples $\left(y, \mathcal{F}_{G}, \beta^{\prime}\right)$, where $y, \mathcal{F}_{G}$ and $\beta^{\prime}$ are as above. Of course, $\mathrm{Fl}_{X}^{\prime}$ is a fibration over $\mathrm{Gr}_{X}^{\prime}$ with the typical fiber $G / B$ and we will denote by $\pi_{X}^{\prime}$ the corresponding projection. Note that our $\mathrm{Gr}_{X}^{\prime}$ is a particular case of the Beilinson-Drinfeld Grassmannian, which was studied in 14 and [4].

The fact that the above functors are indeed representable by indschemes can be proved by a straightforward generalization of the argument that shows that $\mathrm{Gr}$ is representable by an indscheme (cf. Sect. 6). Here is a rough outline of the proof:

Proof. (sketch)

For two integers $m, n \in \mathbb{N}$ we introduce a (relative) Hilbert scheme Hilb ${ }_{n}^{m}$, where for a scheme $S, \operatorname{Hom}\left(S, \operatorname{Hilb}_{n}^{m}\right)$ consists of a map $y: S \rightarrow X$ and a coherent subsheaf $\mathrm{J}$ of

$$
\mathcal{O}^{\oplus n}\left(m \cdot\left(x \times S \cup \Gamma_{y}\right)\right) / \mathcal{O}^{\oplus n}\left(-m \cdot\left(x \times S \cup \Gamma_{y}\right)\right)
$$

over $X \times S$, such that the quotient $\mathcal{O}^{\oplus n}\left(m \cdot\left(x \times S \cup \Gamma_{y}\right)\right) / \mathcal{J}$ is $S$-flat.

For $m^{\prime} \geq m$, there is a natural closed embedding $\mathrm{Hilb}_{n}^{m} \rightarrow \mathrm{Hilb}_{n}^{m^{\prime}}$. It is easy to see that for $G=G L(n), \mathrm{Gr}_{X}^{\prime}$ identifies naturally with the inductive limit $\mathrm{Hilb}_{n}^{m}$.

For general $G$, we choose a faithful representation $G \hookrightarrow G \overrightarrow{L(n)}$ and show as in Sect. A.5 that $\mathrm{Gr}_{X}^{\prime}(G)$ is a closed subfunctor inside $\operatorname{Gr}_{X}^{\prime}\left(G L_{n}\right)$.

3.1.2. Let $\mathrm{Fl}_{X \backslash x}^{\prime}, \mathrm{Fl}_{x}^{\prime}, \mathrm{Gr}_{X \backslash x}^{\prime}$ and $\mathrm{Gr}_{x}^{\prime}$ denote the corresponding subschemes of $\mathrm{Fl}_{X}^{\prime}$ and $\mathrm{Gr}_{X}^{\prime}$, respectively.

Proposition 5. There are natural isomorphisms

$$
\begin{gathered}
\mathrm{Fl}_{X \backslash x}^{\prime} \simeq \mathrm{Gr}_{X \backslash x} \times \mathrm{Fl}, \mathrm{Gr}_{X \backslash x}^{\prime} \simeq \mathrm{Gr}_{X \backslash x} \times \mathrm{Gr} \text { and } \\
\mathrm{Fl}_{x}^{\prime} \simeq \mathrm{Fl}, \mathrm{Gr}_{x}^{\prime} \simeq \mathrm{Gr} .
\end{gathered}
$$


Proof. The fact that $\mathrm{Fl}_{x}^{\prime} \simeq \mathrm{Fl}$ and $\mathrm{Gr}_{x}^{\prime} \simeq \mathrm{Gr}$ follows immediately from Lemma 2. Hence, we must analyze the situation over $X \backslash x$. We will prove the assertion for $\mathrm{Fl}_{X}^{\prime}$, since the proof for $\mathrm{Gr}_{X}^{\prime}$ is the same. We will construct canonical morphisms in both directions between the corresponding functors.

$\Rightarrow$

Let $\left(y, \mathcal{F}_{G}, \beta^{\prime}, \epsilon\right)$ be as above with $\Gamma_{y} \cap x \times S=\emptyset$. First, we define new $G$-bundles $\mathcal{F}_{G}^{1}$ and $\mathcal{F}_{G}^{2}$ as follows:

$\mathcal{F}_{G}^{1}$ (resp., $\mathcal{F}_{G}^{2}$ ) is by definition trivial over $X \times S \backslash \Gamma_{y}$ (resp., $(X \backslash x) \times S$ ) and is identified with $\mathcal{F}_{G}$ over $(X \backslash x) \times S$ (resp., over $\left.X \times S \backslash \Gamma_{y}\right)$.

Since $(X \backslash x) \times S \cup X \times S \backslash \Gamma_{y}=X \times S$, in order to have well-defined $\mathcal{F}_{G}^{1}$ and $\mathcal{F}_{G}^{2}$ over $X \times S$, we must define a gluing data over the intersection $(X \backslash x) \times S \cap X \times S \backslash \Gamma_{y}$. However, the corresponding gluing data for both $\mathcal{F}_{G}^{1}$ and $\mathcal{F}_{G}^{2}$ are provided by the isomorphism $\beta^{\prime}$ : $\left.\left.\mathcal{F}_{G}\right|_{X \times S \backslash\left(x \times S \cup \Gamma_{y}\right)} \rightarrow \mathcal{F}_{G}^{0}\right|_{X \times S \backslash\left(x \times S \cup \Gamma_{y}\right)}$.

By construction, we have the trivializations

$$
\beta^{1}:\left.\left.\mathcal{F}_{G}^{1}\right|_{X \times S \backslash \Gamma_{y}} \rightarrow \mathcal{F}_{G}^{0}\right|_{X \times S \backslash \Gamma_{y}} \text { and } \beta^{2}:\left.\left.\mathcal{F}_{G}^{2}\right|_{(X \backslash x) \times S} \rightarrow \mathcal{F}_{G}^{0}\right|_{(X \backslash x) \times S} .
$$

Since $x \times S \in X \times S \backslash \Gamma_{y}$, the data of $\epsilon$ gives rise to a reduction $\epsilon^{2}$ of $\left.\mathcal{F}_{G}^{2}\right|_{x \times S}$ to $B$.

Thus, to $\left(y, \mathcal{F}_{G}, \beta^{\prime}, \epsilon\right)$ above we attach the point $\left(y, \mathcal{F}_{G}^{1}, \beta^{1}\right) \times\left(\mathcal{F}_{G}^{2}, \beta^{2}, \epsilon^{2}\right) \in \operatorname{Gr}_{X \backslash x} \times \mathrm{Fl}$. $\Leftarrow$

Let $\left(y, \mathcal{F}_{G}^{1}, \beta^{1}\right) \times\left(\mathcal{F}_{G}^{2}, \beta^{2}, \epsilon^{2}\right)$ be an $S$-point of $\mathrm{Gr}_{X \backslash x} \times$ Fl. We attach to it a point of $\mathrm{Fl}_{X}^{\prime}$ as follows:

The $G$-bundle $\mathcal{F}_{G}$ is by definition identified with $\mathcal{F}_{G}^{1}$ over $(X \backslash x) \times S$ and with $\mathcal{F}_{G}^{2}$ over $X \times S \backslash \Gamma_{y}$.

The gluing data for $\mathcal{F}_{G}$ over $X \times S \backslash \Gamma_{y} \cap(X \backslash x) \times S$ is given by the composition:

$$
\left.\left.\left.\mathcal{F}_{G}^{1}\right|_{X \times S \backslash \Gamma_{y} \cap(X \backslash x) \times S} \stackrel{\beta^{1}}{\longrightarrow} \mathcal{F}_{G}^{0}\right|_{X \times S \backslash \Gamma_{y} \cap(X \backslash x) \times S} \stackrel{\beta^{2}}{\longleftarrow} \mathcal{F}_{G}^{2}\right|_{X \times S \backslash \Gamma_{y} \cap(X \backslash x) \times S} .
$$

Thus, we obtain a well-defined $G$-bundle over $X \times S$, which is trivialized, by construction, over $X \times S \backslash \Gamma_{y} \cap(X \backslash x) \times S$.

Finally, the data of $\epsilon^{2}$ for $\mathcal{F}_{G}^{2}$ defines a data of $\epsilon$ for $\mathcal{F}_{G}$, as $\left.\left.\mathcal{F}_{G}\right|_{x \times S} \simeq \mathcal{F}_{G}^{2}\right|_{x \times S}$.

Thus, we have constructed maps $\mathrm{Fl}_{X \backslash x}^{\prime} \leftrightarrows \mathrm{Gr}_{X \backslash x} \times \mathrm{Fl}$ and it is easy to see that they are inverses of one another.

\subsection{The functors $\mathcal{C}(\cdot, \cdot)$.}

3.2.1. According to Sect. 2.1.3 and Proposition 5 we can produce a functor $\mathrm{P}_{\text {Aut }}(\mathrm{Gr}) \times \mathrm{P}(\mathrm{Fl}) \rightarrow$ $\mathrm{P}\left(\mathrm{Fl}_{X \backslash x}^{\prime}\right)$ by

$$
\mathcal{S}, \mathcal{T} \mapsto \mathcal{S}_{X \backslash x} \otimes \mathcal{T}
$$

and we set

$$
\mathcal{C}_{\mathrm{Fl}}(\mathcal{S}, \mathcal{T}):=\Psi_{\mathrm{Fl}_{X}^{\prime}}\left(\mathcal{S}_{X \backslash x} \otimes \mathcal{T}\right)
$$

Thus, $\mathcal{C}_{\mathrm{Fl}}(\cdot, \cdot)$ is a functor $\mathrm{P}_{\mathrm{Aut}}(\mathrm{Gr}) \times \mathrm{P}(\mathrm{Fl}) \rightarrow \mathrm{P}(\mathrm{Fl})$. Analogously, we define the functor $\mathcal{C}_{\mathrm{Gr}}(\cdot, \cdot): \mathrm{P}_{\text {Aut }}(\mathrm{Gr}) \times \mathrm{P}(\mathrm{Gr}) \rightarrow \mathrm{P}(\mathrm{Gr})$ by setting

$$
\mathcal{C}_{\mathrm{Gr}}(\mathcal{S}, \mathcal{T}):=\Psi_{\mathrm{Gr}_{X}^{\prime}}\left(\mathcal{S}_{X \backslash x} \otimes \mathcal{T}\right)
$$

It is easy to see that $\mathrm{Fl}_{X}$ is naturally a closed subscheme of $\mathrm{Fl}_{X}^{\prime}$ : an $S$-point $\left(y, \mathcal{F}_{G}, \beta^{\prime}, \epsilon\right)$ of $\mathrm{Fl}_{X}^{\prime}$ belongs to $\mathrm{Fl}_{X}$ if and only if the trivialization $\beta^{\prime}:\left.\left.\mathcal{F}_{G}\right|_{X \times S \backslash\left(x \times S \cup \Gamma_{y}\right)} \rightarrow \mathcal{F}_{G}^{0}\right|_{X \times S \backslash\left(x \times S \cup \Gamma_{y}\right)}$ extends regularly to $X \backslash \Gamma_{y}$. 
Therefore, we obtain that for $\mathcal{S} \in \mathrm{P}_{\text {Aut }}(\mathrm{Gr})$,

$$
\mathcal{C}_{\mathrm{Fl}}\left(\mathcal{S}, \delta_{1_{\mathrm{Fl}}}\right) \simeq Z(\mathcal{S})
$$

Assertions (a) and (b) of Theorem 11 follow immediately from the following proposition, whose proof will be given in the next section.

Proposition 6. Let $\mathcal{S}$ be an object of $\mathrm{P}_{\text {Aut }}(\mathrm{Gr})$. Then:

(a) For $\mathcal{T} \in \mathrm{P}_{\mathrm{I}}(\mathrm{Fl})$ (resp., $\mathcal{T} \in \mathrm{P}_{G(\widehat{\mathcal{O}})}(\mathrm{Gr})$ ) there is a canonical isomorphism $\mathcal{C}_{\mathrm{Fl}}(\mathcal{S}, \mathcal{T}) \simeq Z(\mathcal{S}) \underset{\mathrm{Fl}}{\mathcal{T}}$ (resp., $\left.\mathcal{C}_{\mathrm{Gr}}(\mathcal{S}, \mathcal{T}) \simeq \mathcal{S}_{\mathrm{Gr}}^{\star} \mathcal{T}\right)$.

(b) For any $\mathcal{T} \in \mathrm{P}(\mathrm{Fl})$ (resp., $\mathcal{T} \in \mathrm{P}(\mathrm{Gr})$ ) and $\mathcal{S} \in \mathrm{P}_{G(\widehat{\mathcal{O}})}(\mathrm{Gr})$ there is a canonical isomorphism

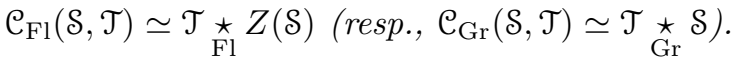

Note that the assertion of Proposition 6 for Gr implies that for $\mathcal{S} \in \mathrm{P}_{G(\widehat{\mathcal{O}})}(\mathrm{Gr}), \mathcal{T} \star \mathcal{G r}$ is perverse for any $\mathcal{T} \in \mathrm{P}(\mathrm{Gr})$ and that if $\mathcal{T}$ is $G(\widehat{\mathcal{O}})$-equivariant too, then $\underset{\mathrm{Gr}}{\star} \mathcal{T} \simeq \mathcal{T} \underset{\mathrm{Gr}}{\star} \mathcal{S}$.

\section{Proofs-I}

\subsection{Some properties of the nearby cycles functor.}

4.1.1. The proof of Theorem 1 i will repeatedly use the following well-known result (cf. 15i)):

Let $y$ be a scheme over $X$ and let $\widetilde{y}$ be another scheme with a proper map $g: \widetilde{y} \rightarrow y$. Let $g_{X \backslash x}$ (resp., $g_{x}$ ) denote the restriction of $g$ to the corresponding subschemes of $\widetilde{y}$.

Theorem 3. There is a natural isomorphism of functors $\mathrm{D}^{b}\left(\widetilde{y}_{X \backslash x}\right) \rightarrow \mathrm{D}^{b}\left(\mathrm{y}_{x}\right)$ :

$$
g_{x !} \circ \Psi_{\tilde{y}} \simeq \Psi_{y} \circ g_{X \backslash x !} .
$$

4.1.2. Let us deduce from Theorem 3 the assertion of of Theorem 1(d).

Proof. We will apply Theorem 3 to the map $\pi_{X}: \mathrm{Fl}_{X} \rightarrow \mathrm{Gr}_{X}$. We have:

$$
\pi_{x !}(Z(\mathcal{S})) \simeq \pi_{!}\left(\Psi_{\mathrm{Fl}_{X}}\left(\mathcal{S}_{X \backslash x} \otimes \delta_{1_{G / B}}\right)\right) \simeq \Psi_{\mathrm{Gr}_{X}}\left(\pi_{X \backslash x !}\left(\mathcal{S}_{X \backslash x} \otimes \delta_{1_{G / B}}\right)\right) .
$$

However, $\pi_{X \backslash x}\left(\mathcal{S}_{X \backslash x} \otimes \delta_{1_{G / B}}\right) \simeq \mathcal{S}_{X \backslash x}$.

Hence, it remains to show that $\Psi_{\mathrm{Gr}_{X}}\left(\mathcal{S}_{X \backslash x}\right) \simeq \mathcal{S}$, i.e. that the vanishing cycles functor $\Phi_{\mathrm{Gr}_{X}}$ applied to $\mathcal{S}_{X}$ yields zero. This follows almost immediately from the fact that $\mathcal{S}_{X}$ was obtained by the twisted external product construction:

Let $Y$ be a closed Aut-invariant subscheme of Gr which contains the support of $\mathcal{S}$. Let $\mathrm{Aut}_{m}$ be a finite dimensional quotient of Aut such that the action of the latter on $Y$ factors through Aut $_{m}$ and let $X_{m}$ be the corresponding Aut ${ }_{m}$-torsor over $X$. Then $Y_{X}:=X_{m} \stackrel{\text { Aut }_{m}}{\times} Y$ is a closed subscheme of $\operatorname{Gr}_{X}$ that contains the support of $\mathcal{S}_{X}$. Hence, it is enough to calculate $\Phi_{Y_{X}}\left(\mathcal{S}_{X}\right)$.

The map $X_{m} \times Y \rightarrow Y_{X}$ is smooth and it is well-known that the functors of nearby and vanishing cycles commute with pull-backs under smooth morphisms. Therefore, it is enough to check that $\Phi_{x_{m} \times Y}$ applied to the pull-back of $\mathcal{S}_{X}$ to $X_{m} \times Y$ is 0 . However, the above pull-back is a direct product $\overline{\mathbb{Q}}_{\ell} x_{m} \otimes \mathcal{S}$. Since the projection $X_{m} \rightarrow X$ is smooth, this implies the required vanishing.

\subsection{Proof of Proposition 6(a).}


4.2.1. To prove Proposition 6(a) we will introduce an auxiliary indscheme $\widetilde{\mathrm{Fl}_{X}^{\prime}}$ over $X$. For a scheme $S, \operatorname{Hom}\left(S, \widehat{\mathrm{Fl}_{X}^{\prime}}\right)$ is the set of 7 -tuples $\left(y, \mathcal{F}_{G}, \mathcal{F}_{G}^{1}, \widetilde{\beta}, \beta^{1}, \epsilon, \epsilon^{1}\right)$, where $\left(y, \mathcal{F}_{G}^{1}, \beta^{1}, \epsilon^{1}\right)$ is a point of $\mathrm{Fl}_{X}, \mathcal{F}_{G}$ is another $G$-bundle, $\widetilde{\beta}$ is an isomorphism $\left.\left.\mathcal{F}_{G}\right|_{(X \backslash x) \times S} \rightarrow \mathcal{F}_{G}^{1}\right|_{(X \backslash x) \times S}$ and $\epsilon$ is a data of a reduction of $\left.\mathcal{F}_{G}\right|_{x \times S}$ to $B$.

By construction, there is a natural projection $p_{X}^{1}: \widetilde{\mathrm{Fl}_{X}^{\prime}} \rightarrow \mathrm{Fl}_{X}$ that "remembers" only the data of $\left(y, \mathcal{F}_{G}^{1}, \beta^{1}, \epsilon^{1}\right)$ and a projection $p_{X}: \widetilde{\mathrm{Fl}_{X}^{\prime}} \rightarrow \mathrm{Fl}_{X}^{\prime}$ that sends $\left(y, \mathcal{F}_{G}, \mathcal{F}_{G}^{1}, \widetilde{\beta}, \beta^{1}, \epsilon, \epsilon^{1}\right)$ to $\left(y, \mathcal{F}_{G}, \beta, \epsilon\right)$, where $\beta$ is the composition $\beta^{1} \circ \widetilde{\beta}$ defined over $X \times S \backslash\left(\Gamma_{y} \cup x \times S\right)$.

By definition, the projection $p_{X}^{1}$ makes $\widetilde{\mathrm{Fl}_{X}^{\prime}}$ a fibration over $\mathrm{Fl}_{X}$ with the typical fiber $\mathrm{Fl}$. Let us make this assertion more precise. Recall that over Gr we had a "tower" of $G(\widehat{\mathcal{O}})_{k}$-torsors $\mathcal{G}_{k}$. An analogous tower exists globally:

We introduce a $\mathrm{I}_{k}$-torsor $\mathcal{G}_{X k}$ over $\mathrm{Fl}_{X}$ that classifies the data of $\left(y, \mathcal{F}_{G}^{1}, \beta^{1}, \epsilon^{1}, \gamma_{k}\right)$, where $\left(y, \mathcal{F}_{G}^{1}, \beta^{1}, \epsilon^{1}\right)$ are as in the definition of $\mathrm{Fl}_{X}$ and $\gamma_{k}$ is a data of a trivialization of $\mathcal{F}_{G}$ on $\mathcal{D}_{k} \times S$, which is compatible with $\epsilon^{1}$ (i.e. the two reductions to $B$ on $S \simeq \mathcal{D}_{0} \times S$ coincide).

Let $Y$ be an I-invariant closed subscheme of $\mathrm{Fl}$ on which $\mathrm{I}$ acts via the quotient $\mathrm{I} \rightarrow \mathrm{I}_{k}$. The fibration $Y_{X}:=\mathcal{G}_{X k} \stackrel{I_{k}}{\times} Y$ over $\mathrm{Fl}_{X}$ associated with the $\mathrm{I}_{k}$-torsor $\mathcal{G}_{X k}$ and the $\mathrm{I}_{k}$-scheme $Y$ is independent of $k$ and is naturally a closed ind-subscheme of $\widetilde{\mathrm{Fl}_{X}^{\prime}}$ (the latter is an inductive limit of indschemes described in the above way).

We will denote by $Y_{x}$ and $Y_{X \backslash x}$ the corresponding subschemes in $Y_{X}$ (note that $Y_{x}$ identifies with the corresponding closed sub-indscheme of the convolution diagram Conv $_{\mathrm{Fl}}$ ).

4.2.2. Let $\mathcal{T}$ be as in Proposition 6(a). Choose $Y$ as above so that $\mathcal{T}$ is supported on $Y$.

As was explained in Sect. 2.2.3, starting from $\mathcal{S} \in \mathrm{P}_{\text {Aut }}(\mathrm{Gr})$, we can form a perverse sheaf $\mathcal{S}_{X \backslash x} \otimes \delta_{1_{G / B}}$ on $\mathrm{Fl}_{X \backslash x}$ and by taking its twisted external product with $\mathcal{T}$ we obtain a perverse sheaf $\left(\mathcal{S}_{X \backslash x} \otimes \delta_{1_{G / B}}\right) \widetilde{\otimes} \mathcal{T}$ on $Y_{X \backslash x}$, and hence on $\widetilde{\mathrm{Fl}_{X}^{\prime}}$.

Let $p_{x}$ and $p_{X \backslash x}$ denote the restriction of the map $p_{X}$ to the corresponding subschemes of $\widetilde{\mathrm{Fl}_{X}^{\prime}}$. The following assertion follows from the definitions:

Lemma 5. (a) The map $Y_{X} \rightarrow \widetilde{\mathrm{Fl}_{X}^{\prime}} \stackrel{p_{X}}{\longrightarrow} \mathrm{Fl}_{X}^{\prime}$ is proper.

(b) The direct image $p_{X \backslash x !}\left(\left(\mathcal{S}_{X \backslash x} \otimes \delta_{1_{G / B}}\right) \widetilde{\otimes} \mathcal{T}\right)$ is canonically isomorphic to the perverse sheaf $\mathcal{S}_{X \backslash x} \otimes \mathcal{T}$ on $\mathrm{Fl}_{X \backslash x}^{\prime}$ constructed in Sect. 3.2.1.

To prove the proposition, let us apply Theorem 3 to the above map $Y_{X} \rightarrow \widetilde{\mathrm{Fl}_{X}^{\prime}} \stackrel{p_{X}}{\longrightarrow} \mathrm{Fl}_{X}^{\prime}$ and the perverse sheaf $\left(\mathcal{S}_{X \backslash x} \otimes \delta_{1_{G / B}}\right) \widetilde{\otimes} \mathcal{T}$ on $Y_{X}$. The map $p_{x}: \widetilde{\mathrm{Fl}_{x}^{\prime}} \rightarrow \mathrm{Fl}_{x}^{\prime}$ identifies with the map $p: \operatorname{Conv}_{F l} \rightarrow \mathrm{Fl}$, therefore, it remains to show that $\Psi_{Y_{X}}\left(\left(\mathcal{S}_{X \backslash x} \otimes \delta_{1_{G / B}}\right) \widetilde{\otimes} \mathcal{T}\right) \simeq Z(\mathcal{S}) \widetilde{\otimes} \mathcal{T}$. The argument is similar to the one we used to prove Theorem 1 $1(\mathrm{~d})$ :

The natural projection $\mathcal{G}_{X k} \times Y \rightarrow \mathcal{G}_{X k} \times \stackrel{I}{k}^{\times} Y:=Y_{X}$ is smooth and has connected fibers. Therefore, it is sufficient to perform the nearby cycles calculation "upstairs", i.e. after the pullback to $\mathfrak{G}_{X k} \times Y$. However, when we pull-back $\left(\mathcal{S}_{X \backslash x} \otimes \delta_{1_{G / B}}\right) \widetilde{\otimes} \mathcal{T}$, it decomposes as a direct product $\left(\mathcal{S}_{X \backslash x} \otimes \delta_{1_{G / B}}\right) \otimes \mathcal{T}$, where $\left(\mathcal{S}_{X \backslash x} \otimes \delta_{1_{G / B}}\right)_{k}$ denotes the pull-back of the perverse sheaf $\mathcal{S}_{X \backslash x} \otimes \delta_{1_{G / B}}$ from $\mathrm{Fl}_{X \backslash x}$ to $\mathcal{G}_{X \backslash x k}$.

Let $Z(\mathcal{S})_{k}$ denote the pull-back of $Z(\mathcal{S})$ under the map $\mathcal{G}_{x k} \rightarrow \mathrm{Fl}_{x} \simeq \mathrm{Fl}$. We have: $\Psi_{\mathcal{G}_{X k}}\left(\mathcal{S}_{X \backslash x} \otimes \delta_{1_{G / B}}\right)_{k} \simeq Z(\mathcal{S})_{k}$, since the map $\mathcal{G}_{X k} \rightarrow \mathrm{Fl}_{X}$ is smooth. Hence,

$$
\Psi_{\mathcal{G}_{X} \times Y}\left(\left(\mathcal{S}_{X \backslash x} \otimes \delta_{1_{G / B}}\right)_{k} \otimes \mathcal{T}\right) \simeq Z(\mathcal{S})_{k} \otimes \mathcal{T},
$$

which is what we had to prove. 
The proof of Proposition 6(a) for Gr is completely similar (and even simpler).

\subsection{Proof of Proposition 6(b).}

4.3.1. To prove Proposition 6 (b) we will introduce another scheme $\widetilde{\mathrm{Fl}_{X}^{\prime}}$, different from the one of the previous subsection. (We are going to prove Proposition 6(b) for Fl, since the argument for $\mathrm{Gr}$ is the same).

The new $\widetilde{\mathrm{Fl}_{X}^{\prime}}$ classifies 7 -tuples $\left(y, \mathcal{F}_{G}, \mathcal{F}_{G}^{1}, \widetilde{\beta}, \beta^{1}, \epsilon, \epsilon^{1}\right)$, where $\left(\mathcal{F}_{G}^{1}, \beta^{1}, \epsilon^{1}\right)$ is an $S$-point of $\mathrm{Fl}$ (in particular, $\beta^{1}$ is a trivialization of $\mathcal{F}_{G}^{1}$ on $\left.(X \backslash x) \times S\right), \mathcal{F}_{G}$ is another $G$-bundle, $y$ is another $S$-point of $X, \widetilde{\beta}$ is an isomorphism $\left.\left.\mathcal{F}_{G}\right|_{X \times S \backslash \Gamma_{y}} \rightarrow \mathcal{F}_{G}^{1}\right|_{X \times S \backslash \Gamma_{y}}$ and $\epsilon$ is a data of a reduction of $\left.\mathcal{F}_{G}\right|_{x \times S}$ to $B$.

We have the projections $p_{X}^{1}$ and $p_{X}$ from $\widetilde{\mathrm{Fl}_{X}^{\prime}}$ to $\mathrm{Fl}$ and $\mathrm{Fl}_{X}^{\prime}$, respectively: $p_{X}^{1}$ remembers the quadruple $\left(\mathcal{F}_{G}^{1}, \beta^{1}, \epsilon^{1}\right)$ and $p_{X}$ sends the above 7 -tuple to $\left(y, \mathcal{F}_{G}, \beta, \epsilon\right)$, where $\beta$ is the composition $\beta^{1} \circ \widetilde{\beta}$ defined over $X \times S \backslash\left(\Gamma_{y} \cup x \times S\right)$.

Remark. The essential difference between points (a) and (b) of the proposition is that in the latter case, $\mathrm{Fl}_{X}^{\prime}$ is not strictly speaking a fibration over $\mathrm{Fl}_{X}$ attached to a group in the sense of Sect. 0.2. For that reason we have to work harder.

Let $\widetilde{\mathrm{Fl}_{X \backslash x}^{\prime}}$ and $\widetilde{\mathrm{Fl}_{x}^{\prime}}$ denote the corresponding subschemes of $\widetilde{\mathrm{Fl}_{X}^{\prime}}$. First, observe that $\widetilde{\mathrm{Fl}_{x}^{\prime}}$ again identifies canonically with Conv $\mathrm{Fl}$. Secondly, $\mathrm{Fl}_{X \backslash x}^{\prime}$ is naturally a closed subscheme in $\widehat{\mathrm{Fl}_{X \backslash x}^{\prime}}$ :

Indeed to an $S$-point $\left(y, \mathcal{F}_{G}, \beta^{\prime}, \epsilon\right)$ of $\mathrm{Fl}_{X \backslash x}^{\prime}$ we attach the data of $\left(y, \mathcal{F}_{G}, \mathcal{F}_{G}^{1}, \widetilde{\beta}, \beta^{1}, \epsilon, \epsilon^{1}\right)$, where $\left(y, \mathcal{F}_{G}, \epsilon\right)$ are with no change, $\mathcal{F}_{G}^{1}$ is set to be isomorphic to $\mathcal{F}_{G}$ over $X \times S \backslash \Gamma_{y}$ and to $\mathcal{F}_{G}^{0}$ over $(X \backslash x) \times S$ (with the gluing data provided by $\beta^{\prime}$ ), $\epsilon^{1}$ being induced by $\epsilon$ and $\widetilde{\beta}, \beta^{1}$ coming by construction.

Thus, for $\mathcal{S} \in \mathrm{P}_{G(\widehat{\mathcal{O}})}(\mathrm{Gr})$ and $\mathcal{T} \in \mathrm{P}(\mathrm{Fl})$, by taking the direct image of $\mathcal{S}_{X \backslash x} \otimes \mathcal{T} \in \mathrm{P}\left(\mathrm{Fl}_{X \backslash x}^{\prime}\right)$, we obtain a perverse sheaf $\left(\mathcal{S}_{X \backslash x} \otimes \mathcal{T}\right) \sim$ on $\widetilde{\mathrm{Fl}_{X \backslash x}^{\prime}}$. Its direct image under $p_{X \backslash x}: \widetilde{\mathrm{Fl}_{X \backslash x}^{\prime}} \rightarrow \mathrm{Fl}_{X \backslash x}^{\prime}$ is canonically isomorphic to $\mathcal{S}_{X \backslash x} \otimes \mathcal{T}$.

By applying Theorem 3 to $\left(\mathcal{S}_{X \backslash x} \otimes \mathcal{T}\right)^{\sim}$, we conclude that it is enough to show that

$$
\Psi_{\widetilde{\mathrm{Fl}_{X}^{\prime}}}\left(\left(\mathcal{S}_{X \backslash x} \otimes \mathcal{T}\right)^{\sim}\right) \simeq \mathcal{T} \widetilde{\otimes} Z(\mathcal{S})
$$

4.3.2. Let $Y$ be the support of $\mathcal{T}$ in Fl. We can replace our initial $X$ by $X=\mathbb{A}^{1}$ and in the latter case there exists an étale and surjective map $\mathcal{U} \rightarrow Y$ such that when we pull-back the universal $G$-bundle from $X \times \mathrm{Fl}$ to $X \times \mathcal{U}$, it becomes trivial. f Let $\phi_{\mathfrak{u}}^{\prime}$ be a trivialization; by further localizing $\mathcal{U}$, we can arrange that the two reductions to $B$ of our $G$-bundle on $x \times \mathcal{U}$ (one coming from $\phi_{u}^{\prime}$ and the other from the universal property of $\mathrm{Fl}$ ) coincide.

Let us make a base change $\widetilde{\mathrm{Fl}_{X}^{\prime}} \Rightarrow \widetilde{\mathrm{Fl}_{X}^{\prime}} \times \underset{\mathrm{Fl}}{\mathcal{U}}$. Since the projection $\widetilde{\mathrm{Fl}_{X}^{\prime}} \times \underset{\mathrm{Fl}}{\mathcal{U}} \rightarrow \mathrm{Fl}_{X}^{\prime}$ is étale over the support of $\left(\mathcal{S}_{X \backslash x} \otimes \mathcal{T}\right)^{\sim}$, it is enough to perform the nearby cycles calculation "upstairs". Namely, let $\left(\mathcal{S}_{X \backslash x} \otimes \mathcal{T}\right) \tilde{u}$ and $(\mathcal{T} \widetilde{\nabla} Z(\mathcal{S}))_{u}$ denote the pull-backs of $\left(\mathcal{S}_{X \backslash x} \otimes \mathcal{T}\right) \sim$ and $\mathcal{T} \widetilde{\nabla} Z(\mathcal{S})$ to $\widetilde{\mathrm{Fl}_{X \backslash x}^{\prime}} \times \underset{\mathrm{Fl}}{\mathcal{U}}$ and $\widetilde{\mathrm{Fl}_{x}^{\prime}} \underset{\mathrm{Fl}}{\times \mathcal{U}}$, respectively.

We must show that

$$
\Psi_{\widetilde{\mathrm{Fl}_{X}^{\prime}} \times \mathrm{Fl}}\left(\left(\mathcal{S}_{X \backslash x} \otimes \mathcal{T}\right) \widetilde{u}\right) \simeq(\mathcal{T} \widetilde{\nabla} Z(\mathcal{S})) u
$$

\footnotetext{
${ }^{5}$ Indeed, according to [9], we can find $\mathcal{U}$, étale and surjective over $Y$, such that our $G$-bundle on $X \times \mathcal{U}$ admits a reduction to $B$. However, it is well-known that any $B$-bundle on $\mathbb{A}^{1} \times \mathcal{U}$ is pulled back from $\mathcal{U}$. Hence, by localizing even more with respect to $\mathcal{U}$, we can make this $B$-bundle trivial.
} 
and that this isomorphism is independent of the choice of the trivialization $\phi_{\mathfrak{u}}^{\prime}$.

Notice now, that the choice of $\phi_{\mathcal{U}}^{\prime}$ defines an identification $\widetilde{\mathrm{Fl}_{X}^{\prime}} \underset{\mathrm{Fl}}{\times} \times \mathcal{U} \simeq \mathrm{Fl}_{X} \times \mathcal{U}$. Indeed, since the univesral bundle $\mathcal{F}_{G}^{\prime}$ over $X \times \mathcal{U}$ is trivial, the data of $\widetilde{\beta}$ is equivalent to the trivialization of $\mathcal{F}_{G}$ off $\Gamma_{y}$.

However, when we restrict the universal bundle to $(X \backslash x) \times \mathcal{U}$, it has two different trivializations! One comes from $\phi_{\mathfrak{u}}^{\prime}$ and the other from the fact that the universal bundle on $X \times \mathrm{Fl}$ is by definition trivialized over $(X \backslash x) \times \mathcal{U}$. These trivializations differ by a map $\phi_{\mathfrak{U}}:(X \backslash x) \times \mathcal{U} \rightarrow G$.

Correspondingly, we have two different closed embeddings of $\operatorname{Gr}_{X \backslash x} \times \mathcal{U}$ into $\widetilde{\mathrm{Fl}_{X \backslash x}^{\prime}} \times \underset{\mathrm{Fl}}{ } \times$ :

Embedding (1) is the composition of the above identification $\widetilde{\mathrm{Fl}_{X}^{\prime}} \underset{\mathrm{Fl}}{\mathcal{U}} \simeq \mathrm{Fl}_{X} \times \mathcal{U}$ and the embedding $\mathrm{Gr}_{X \backslash x}=\mathrm{Gr}_{X \backslash x} \times 1_{G / B} \hookrightarrow \mathrm{Fl}_{X \backslash x}$ of Proposition 3.

Embedding (2) comes from the embedding $\mathrm{Fl}_{X \backslash x}^{\prime} \rightarrow \mathrm{Fl}_{X \backslash x}^{\prime}$ described in Sect. 4.3.1 and the isomorphism $\mathrm{Fl}_{X \backslash x}^{\prime} \simeq \mathrm{Gr}_{X \backslash x} \times \mathrm{Fl}$ of Proposition 5 .

It is easy to see that these two embeddings differ by the automorphism of $\mathrm{Gr}_{X \backslash x} \times \mathcal{U}$ induced by $\phi_{u}$ as in Proposition 2 .

By construction, $\left(\mathcal{S}_{X \backslash x} \otimes \mathcal{T}\right) \tilde{\mathcal{u}}$ is isomorphic to the direct image under the above Embedding (2) of $\mathcal{S}_{X \backslash x} \otimes \mathcal{T}_{\mathcal{U}}$ (here $\mathcal{T}_{\mathcal{U}}$ is the pull-back of $\mathcal{T}$ under $\mathcal{U} \rightarrow \mathrm{Fl}$ ). Hence, by Proposition 2, it is isomorphic also to the direct image of the same $\mathcal{S}_{X \backslash x} \otimes \mathcal{T}_{\mathcal{U}}$ under Embedding (1).

Hence, on the one hand,

$$
\Psi_{\widetilde{\mathrm{Fl}_{X}^{\prime}} \times \mathfrak{\mathrm { Fl }}}\left(\left(\mathcal{S}_{X \backslash x} \otimes \mathcal{T}\right) \tilde{\mathcal{U}}\right) \simeq \Psi_{\mathrm{Fl}_{X} \times \mathfrak{U}}\left(\left(\mathcal{S}_{X \backslash x} \otimes \delta_{1_{G / B}}\right) \otimes \mathcal{T}_{\mathfrak{U}}\right) \simeq Z(\mathcal{S}) \otimes \mathcal{T}_{\mathfrak{U}}
$$

But on the one hand, under the identification $\widetilde{\mathrm{Fl}_{x}^{\prime}} \underset{\mathrm{Fl}}{\times} \mathcal{U} \simeq \mathrm{Fl} \times \mathcal{U}$, the complex $(\mathcal{T} \widetilde{\otimes} Z(\mathcal{S})) u$ goes over to the same $Z(\mathcal{S}) \otimes \mathcal{T}_{\mathcal{U}}$.

This proves the existence of the required isomorphism. Let us now analyze what happens when we modify $\phi_{\mathcal{U}}^{\prime}$ by a map $\phi_{\mathcal{U}}^{\prime \prime}: X \times \mathcal{U} \rightarrow G\left(\phi_{\mathcal{U}}^{\prime \prime}\right.$ must send $x \times \mathcal{U}$ to $\left.B \subset G\right)$. The effect would be the automorphism of $\mathrm{Fl}_{X} \times \mathcal{U}$ induced by $\phi_{\mathcal{U}}^{\prime \prime}$, as in Proposition 2. This does not change the identification $\Psi_{\widetilde{\mathrm{Fl}_{X}^{\prime}} \times \mathrm{Fl}}\left(\left(\mathcal{S}_{X \backslash x} \otimes \mathcal{T}\right) \tilde{\mathcal{U}}\right) \simeq(\mathcal{T} \widetilde{\otimes} Z(\mathcal{S})) u$, by the definition of the I-equivariant structure on $Z(\mathcal{S})$ (cf. proof of Proposition 4 ).

\section{Proofs-II}

\subsection{The monodromy action.}

5.1.1. Recall the situation of Sect. 2.2.2. Let $\Gamma$ (resp., $\Gamma^{g}$ ) denote the full (resp., geometric) Galois group that corresponds to the pair $x \in X$. In other words, $\Gamma$ (resp., $\Gamma^{g}$ ) is the Galois group of the field of fractions of the henselization (resp., strict henselization) of the local ring $\mathcal{O}_{x}$. As was mentioned in Sect. 2.2.2, we are fixing a splitting $\Gamma \simeq \Gamma^{g} \rtimes \operatorname{Gal}\left(\overline{\mathbb{F}}_{q} / \mathbb{F}_{q}\right)$. There is a canonical homomorphism $\Gamma^{g} \stackrel{t_{\ell}}{\rightarrow} \mathbb{Z}_{\ell}(1)$ (we are taking into account the action of $\operatorname{Gal}\left(\overline{\mathbb{F}}_{q} / \mathbb{F}_{q}\right)$ on $\Gamma^{g}$ and $\left.\mathbb{Z}_{\ell}(1)\right)$.

Let $\rho: \Gamma \rightarrow \operatorname{Aut}(V)$ be a (continuous) representation. Following [10], there exists a canonical nilpotent endomorphism $M_{V}: V \rightarrow V(-1)$ and a subgroup $\Gamma^{\prime} \subset \Gamma^{g}$ of finite index, such that for any $\gamma \in \Gamma^{\prime}$

$$
\rho(\gamma)=\exp \left(t_{\ell}(\gamma) \cdot M_{V}\right): V \rightarrow V
$$


Recall that a representation $(\rho, V)$ is called unipotent if $\Gamma^{\prime}=\Gamma^{g}$. In this case, there exists a $\Gamma$-stable filtration on $V$ such that the action of $\Gamma^{g}$ on the successive quotients is trivial.

In general, any representation $\rho: \Gamma \rightarrow \operatorname{Aut}(V)$ can be decomposed as a direct $\operatorname{sum} V=$ $V^{u n} \oplus V^{\text {non-un }}$, where $V^{u n}$ is unipotent and $V^{n o n-u n}$ is purely non-unipotent (i.e. every irreducible subquotient of $V^{n o n-u n}$ is non-trivial as a $\Gamma^{g}$-representation).

Now, the basic property of the functor $\Psi_{y}$ is that it carries the action of $\Gamma$. In particular, for $\mathcal{S} \in \mathrm{P}\left(y_{X \backslash x}\right)$, we have a nilpotent endomorphism $M_{\mathcal{S}}: \Psi_{y}(\mathcal{S}) \rightarrow \Psi_{y}(\mathcal{S})(-1)$ and a decomposition $\Psi_{y}(\mathcal{S}) \simeq \Psi_{y}^{u n}(\mathcal{S}) \oplus \Psi_{y}^{n o n-u n}(\mathcal{S})$.

Lemma 6. The isomorphism of functors given by Theorem 3 respects the action of $\Gamma$.

5.1.2. Let us apply the above discussion to the situation $y=\mathrm{Fl}_{X}$. We obtain for every $\mathcal{S} \in \mathrm{P}_{G(\widehat{\mathcal{O}})}(\mathrm{Gr})$ an endomorphism $M_{\mathcal{S}}: Z(\mathcal{S}) \rightarrow Z(\mathcal{S})(-1)$ and a canonical decomposition $Z(\mathcal{S})=Z(\mathcal{S})^{\text {un }} \oplus Z(\mathcal{S})^{\text {non }- \text { un }}$.

The following is not essential for our purposes, but is important as an observation:

Proposition 7. $Z(\mathcal{S})^{\text {non }- \text { un }}=0$.

Proof. First, by the construction of the functor $\mathcal{C}_{\mathrm{Fl}}(\cdot, \cdot)$, we have a $\Gamma$-action on $\mathcal{C}_{\mathrm{Fl}}(\mathcal{S}, \mathcal{T})$ for every $\mathcal{T} \in \mathrm{P}(\mathrm{Fl})$. Moreover, Lemma 6 implies that the isomorphisms $\mathcal{C}_{\mathrm{Fl}}(\mathcal{S}, \mathcal{T}) \simeq \mathcal{T} \star Z(\mathcal{S})$ and $\mathcal{C}_{\mathrm{Fl}}(\mathcal{S}, \mathcal{T}) \simeq Z(\mathcal{S}) \underset{\text { Fl }}{\star} \mathcal{T}\left(\right.$ for $\mathcal{T} \in \mathrm{P}_{\mathrm{I}}(\mathrm{Fl})$ ) are compatible with this $\Gamma$-action.

Therefore, for $\mathcal{T} \in \mathrm{P}_{\mathrm{I}}(\mathrm{Fl})$ we have the isomorphisms

$$
Z(\mathcal{S})^{u n} \underset{\mathrm{Fl}}{\star} \mathcal{T} \simeq \mathcal{T}{ }_{\mathrm{Fl}} \underset{(\mathcal{S})^{u n}}{\text { and }} Z(\mathcal{S})^{\text {non-un }} \underset{\mathrm{Fl}}{\star} \mathcal{T} \simeq \underset{\mathrm{Fl}}{\star} Z(\mathcal{S})^{\text {non-un }} .
$$

In addition, Lemma 6 applied to the map $\pi_{X}: \mathrm{Fl}_{X} \rightarrow \operatorname{Gr}_{X}$ implies that $\pi_{!}\left(Z(\mathcal{S})^{\text {non-un }}\right) \simeq$ $\Psi_{\mathrm{Gr}_{X}}^{\text {non-un }}\left(S_{X \backslash x}\right)=0$.

Let us study the function corresponding to $Z(\mathcal{S})^{\text {non-un }}$ on $\mathrm{Fl}\left(\mathbb{F}_{q}\right)$. (By enlarging the finite field we may assume that $G$ is split.)

The fact that $Z(\mathcal{S})^{\text {non-un }} \star \mathcal{T}$ and $\mathcal{T} \star Z(\mathcal{F l})^{\text {non-un }}$ are isomorphic as perverse sheaves defined over $\mathbb{F}_{q}$ for any $\mathcal{T} \in \mathrm{P}_{\mathrm{I}}(\mathrm{Fl})$, implies that the corresponding element of $\mathbf{H}_{\mathrm{I}}$ is central. At the same time, it vanishes under the map $\pi: \mathbf{H}_{\mathrm{I}} \rightarrow \mathbf{H}_{s p h}$. Hence, this function is zero, as $\pi$ induces an isomorphism $Z\left(\mathbf{H}_{\mathrm{I}}\right) \rightarrow \mathbf{H}_{s p h}$, by Bernstein's theorem.

Now, the same fact is true not only for $\mathbb{F}_{q}$, but also for all finite field extensions $\mathbb{F}_{q} \subset \mathbb{F}_{q^{\prime}}$, which implies that $Z(\mathcal{S})^{\text {non-un }}=0$.

Remark. The above proof of Proposition 7 uses the "faisceaux-fonctions" correspondence and Bernstein's theorem. In fact, it is not difficult to give a purely geometric proof, which we will do elsewhere.

\subsection{Proof of Theorem 1 $1(\mathbf{c})$.}

5.2.1. The proof will rely on the following general property of the nearby cycles functor, proved in [2]:

Now let $y^{1}$ and $y^{2}$ be two schemes mapping to $X$ and let $\mathcal{S}^{1}$ and $\mathcal{S}^{2}$ be objects in $\mathrm{D}^{b}\left(y_{X \backslash x}^{1}\right)$ and $\mathrm{D}^{b}\left(y_{X \backslash x}^{2}\right)$, respectively. We will denote by $\mathcal{S}^{1} \underset{X}{\otimes} \mathcal{S}^{2}$ the $*$-restriction of $\mathcal{S}^{1} \otimes \mathcal{S}^{2}$ to ${ }_{X \backslash x}^{y_{X \backslash x}} \underset{X \backslash x}{y^{2}}$, cohomologically shifted by 1 to the right. 
Theorem 4. There is a canonical isomorphism in $\mathrm{D}^{b}\left(y_{x}^{1} \times y_{x}^{2}\right)$ :

$$
\Psi_{y^{1} \times y^{2}}\left(\mathcal{S}^{1} \underset{X}{\otimes} \mathcal{S}^{2}\right) \simeq \Psi_{y^{1}}\left(\mathcal{S}^{1}\right) \otimes \Psi_{y^{2}}\left(\mathcal{S}^{2}\right) .
$$

Moreover, this isomorphism is compatible with the $\Gamma$-action.

5.2.2. To prove Theorem 1 1 (c), we introduce the schemes $\operatorname{Conv}_{X}$ and $\operatorname{Conv}_{X}^{\prime}$ over $X$. The scheme $\operatorname{Conv}_{X}$ is by definition $X \underset{\text { Aut }}{\times} \operatorname{Conv}_{\mathrm{Gr}}$. In other words, $\operatorname{Hom}\left(S, \operatorname{Conv}_{X}\right)$ is the set of quintuples $\left(y, \mathcal{F}_{G}, \widetilde{\beta}, \mathcal{F}_{G}^{1}, \beta^{1}\right)$, where $\left(y, \mathcal{F}_{G}^{1}, \beta^{1}\right)$ is a point of $\mathrm{Gr}_{X}, \mathcal{F}_{G}$ is another $G$-bundle over $X \times S$ and $\widetilde{\beta}$ is an isomorphism $\left.\left.\mathcal{F}_{G}\right|_{X \times S \backslash \Gamma_{y}} \simeq \mathcal{F}_{G}^{1}\right|_{X \times S \backslash \Gamma_{y}}$.

For $\operatorname{Conv}_{X}^{\prime}$, we put $\operatorname{Hom}\left(S, \operatorname{Conv}_{X}^{\prime}\right)$ to be the set of 7 -tuples $\left(y, \mathcal{F}_{G}, \widetilde{\beta}, \epsilon, \mathcal{F}_{G}^{1}, \beta^{1}, \epsilon^{1}\right)$, where $\left(y, \mathcal{F}_{G}, \widetilde{\beta}, \mathcal{F}_{G}^{1}, \beta^{1}\right)$ are as above and $\epsilon$ (resp., $\left.\epsilon^{1}\right)$ is a reduction to $B$ of $\left.\mathcal{F}_{G}\right|_{x \times S}$ (resp., of $\left.\mathcal{F}_{G}^{1}\right|_{x \times S}$ ).

Let $\operatorname{Conv}_{X \backslash x}, \operatorname{Conv}_{x}, \operatorname{Conv}_{X \backslash x}^{\prime}$ and $\operatorname{Conv}_{x}^{\prime}$ denote the corresponding subschemes of Conv $X$ and $\mathrm{Conv}_{X}^{\prime}$, respectively. Let also $p_{X}$ and $p_{X}^{1}$ denote the standard projections from $\operatorname{Conv}_{X}^{\prime}$ to $\mathrm{Fl}_{X}$.

Lemma 7. We have natural identifications

$$
\operatorname{Conv}_{X \backslash x}^{\prime} \simeq \operatorname{Conv}_{X \backslash x} \times G / B \times G / B \text { and } \operatorname{Conv}_{x}^{\prime} \simeq \operatorname{Conv}_{\mathrm{Fl}} .
$$

Thus, starting from two objects $\mathcal{S}^{1}$ and $\mathcal{S}^{2}$ of $\mathrm{P}_{G(\widehat{\mathcal{O}})}(\mathrm{Gr})$, we can construct a perverse sheaf $\mathcal{S}_{X \backslash x}^{1} \widetilde{\otimes} \delta_{X \backslash x}^{2} \otimes \delta_{1_{G / B}} \otimes \delta_{1_{G / B}}$ on $\operatorname{Conv}_{X \backslash x}^{\prime}$. By applying Theorem 3 to $p_{X}: \operatorname{Conv}_{X}^{\prime} \rightarrow \mathrm{Fl}_{X}$, we obtain that in order to prove Theorem 1 (c) and Theorem 2, we must verify the following:

There exists a $\Gamma$-equivariant isomorphism

$$
\Psi_{\mathrm{Conv}_{X}^{\prime}}\left(\mathcal{S}_{X \backslash x}^{1} \widetilde{\nabla} \mathcal{S}_{X \backslash x}^{2} \otimes \delta_{1_{G / B}} \otimes \delta_{1_{G / B}}\right) \simeq Z\left(\mathcal{S}^{1}\right) \widetilde{\otimes} Z\left(\mathcal{S}^{2}\right) \in \mathrm{P}\left(\operatorname{Conv}_{\mathrm{Fl}}\right) .
$$

This proof of this statement is a variation of the argument presented in the proof of Proposition 6 (b). We allow ourselves to be more sketchy:

5.2.3. Let $Y$ be the support of $\mathcal{S}_{X \backslash x}^{1} \bigotimes \delta_{1_{G / B}}$ in $\mathrm{Fl}_{X}$. As in the proof of Proposition 6(b), we can assume that there exists a surjective étale map $\mathcal{U} \rightarrow Y$ with the property that the pull-back of the universal $G$-bundle from $X \times \mathrm{Fl}_{X}$ to $X \times \mathcal{U}$ is trivial. Let us fix a trivialization compatible with the existing $B$-structure on $x \times \mathcal{U}$. Let $\mathcal{U}_{X \backslash x}$ and $\mathcal{U}_{x}$ denote the corresponding subschemes of $\mathcal{U}$.

As before, it sufficient to carry out the nearby cycles calculation on $\mathcal{U}_{\mathrm{Fl}_{X}} \times \operatorname{Conv}_{X}^{\prime}$, where the fiber product is defined using the projection $p_{X}^{1}: \operatorname{Conv}_{X}^{\prime} \rightarrow \mathrm{Fl}_{X}$.

Let $\mathcal{E}\left(\mathcal{S}^{1}, \mathcal{S}^{2}\right)$ denote the pull-back of the perverse sheaf $\mathcal{S}_{X \backslash x}^{1} \widetilde{\otimes} \mathcal{S}_{X \backslash x}^{2} \otimes \delta_{1_{G / B}} \otimes \delta_{1_{G / B}}$ from $\operatorname{Conv}_{X \backslash x}^{\prime}$ to $\mathcal{U}_{X \backslash x} \underset{\mathrm{Fl}_{X \backslash x}}{\times} \operatorname{Conv}_{X \backslash x}^{\prime}$. Let $\mathcal{E}\left(\mathcal{S}^{1}\right)_{X \backslash x}$ (resp., $\left.\mathcal{E}\left(\mathcal{S}^{1}\right)_{x}\right)$ denote the pull-back of $\mathcal{S}_{X \backslash x}^{1} \nabla$ $\delta_{1_{G / B}}$ (resp., of $\left.Z\left(\mathcal{S}_{1}\right)\right)$ to $\mathcal{U}_{X \backslash x}$ (resp., to $\mathcal{U}_{x}$ ).

As in the proof of Proposition 6(b), the trivialization of the pulled-back universal $G$-bundle on $X \times \mathcal{U}$ defines an isomorphism $\underset{\mathrm{Fl}_{X}}{\mathfrak{X}} \mathrm{Conv}_{X}^{\prime} \simeq \mathcal{U} \underset{X}{\times \mathrm{Fl}_{X}}$. Moreover, under this isomorphism the perverse sheaf $\mathcal{E}\left(\mathcal{S}^{1}, \mathcal{S}^{2}\right)$ becomes identified with $\mathcal{E}\left(\mathcal{S}^{1}\right)_{X \backslash x} \underset{X}{\underset{X}{\otimes}}\left(\mathcal{S}_{X \backslash x}^{2} \otimes \delta_{1_{G / B}}\right)$.

Therefore, on the one hand, using Theorem $\mathbb{A}$, we obtain

$$
\Psi_{\mathfrak{F}_{X} \times \operatorname{Conv}_{X}^{\prime}}\left(\mathcal{E}\left(\mathcal{S}^{1}, \mathcal{S}^{2}\right)\right) \simeq \Psi_{\mathcal{u}}\left(\mathcal{E}\left(\mathcal{S}^{1}\right)_{X \backslash x}\right) \otimes \Psi_{\mathrm{Fl}_{X}}\left(\mathcal{S}_{X \backslash x}^{2} \otimes \delta_{1_{G / B}}\right) \simeq \mathcal{E}\left(\mathcal{S}^{1}\right)_{x} \otimes Z\left(\mathcal{S}_{2}\right) .
$$

On the other hand, the pull-back of $Z\left(\mathcal{S}_{1}\right) \widetilde{\otimes} Z\left(\mathcal{S}_{2}\right)$ under $\mathcal{U}_{x} \times \mathrm{Fl}_{x} \rightarrow$ Conv $_{\mathrm{Fl}}$ identifies also with $\mathcal{E}\left(\mathcal{S}^{1}\right)_{x} \otimes Z\left(\mathcal{S}_{2}\right)$, which is what we had to prove. 


\section{ApPEndix}

A.1. Let $F, F^{\prime}$ be two countravariant functors Schemes $\rightarrow$ Sets and let $F^{\prime} \rightarrow F$ be a morphism. We say that $F^{\prime}$ is a closed subfunctor of $F$ if for any scheme $S$ and any $f_{S} \in F(S)$ the Cartesian product functor $\underline{S} \underset{F}{\times} F^{\prime}$ is representable by a closed subscheme of $S$.

Let $Y_{1} \hookrightarrow Y_{2} \hookrightarrow \ldots \hookrightarrow Y_{n} \hookrightarrow \ldots$ be a directed system of schemes, where all maps $Y_{i} \hookrightarrow Y_{i+1}$ are closed embeddings.

We define a functor $Y_{i}$ on the category of schemes by setting $\operatorname{Hom}\left(S, Y_{i}\right):=\operatorname{Hom}\left(S, Y_{i}\right)$ for $S$ quasi-compact, and by extending it to all schemes by requiring that it is a sheaf in Zarisky topology.

A (strict) indscheme is, by definition, a functor $F$ which is isomorphic to some $Y_{i}$ as above. We say that an indscheme is of ind-finite type if the above family of $Y_{i}$ 's can be chosen in such a way that all of them are of finite type.

In what follows we will work with indschemes of ind-finite type only. The basic objects of this paper, that is $\mathrm{Gr}_{X}, \mathrm{Fl}, \mathrm{Gr}_{X}$, etc., all have this property. The only indscheme not of ind-finite type that appears in this paper is $G(\widehat{\mathcal{K}})$, but it has been used only as a functor. Thus, unless specified otherwise, by an indscheme we will mean an indscheme of ind-finite type.

Lemma 8. Let $F \simeq Y_{i}$ be an indscheme. Then:

(a) If $Z$ is a scheme of finite type and $\underline{Z} \rightarrow F$ is a closed subfunctor, then there exists an index $i$ such that $Z$ is a closed subscheme of $Y_{i}$. (In this case we will say that $Z$ is a closed subscheme of $F$.)

(b) If $F^{\prime}$ is a closed subfunctor of $F$, then $F^{\prime}$ is an indscheme too.

(c) If $F$ is isomorphic to $Y_{i}^{\prime}$ for a different family of schemes $Y_{i}^{\prime}$, then for every $i_{1}$ there exists an $i_{2}$ such that $Y_{i_{1}}^{\prime}$ is a closed subscheme of $Y_{i_{2}}$ and vice versa.

The proof is a tautology.

A.2. For an indscheme $F=Y_{i}$ we define the category of perverse sheaves on it as $\mathrm{P}(F):=$ $\mathrm{P}\left(Y_{i}\right)$, where the functors $\mathrm{P}\left(\overrightarrow{Y_{i}}\right) \rightarrow \mathrm{P}\left(Y_{i+1}\right)$ are, of course, the direct image functors. This is $\overrightarrow{\text { again }}$ an abelian category, since the "direct image under a closed embedding" functor is exact. Similarly, one can define the derived category $\mathrm{D}^{b}(F):=\mathrm{D}^{b}\left(Y_{i}\right)$, which is a triangulated category due to the exactness property mentioned above. Actually, we do no need derived categories in this paper and we discuss them only for the sake of completeness.

Point (3) of Lemma 8 implies that these definitions do not depend on the choice of a presentation of $F$ as $Y_{i}$, i.e. $\mathrm{P}(F)$ and $\mathrm{D}^{b}(F)$ are intrinsically attached to $F$.

We emphasize again, that a perverse sheaf on an indscheme is by definition supported on a closed subscheme of finite type. This means that this notion is essentially "finite-dimentional".

A.3. Let $H$ be a group-scheme, which is a projective limit of linear algebraic groups: $H=H_{k}$.

The basic examples are $H=G(\widehat{\mathcal{O}}), H=$ Aut.

Let $F$ be an indscheme and let $\underline{H}$ act on $F$ (in the sense of functors). We say that this action is "nice" if the following holds: every closed subscheme $Z$ of $F$ is contained in a larger closed subscheme $Z^{\prime}$ with the property that $Z^{\prime}$ is $H$-stable and the action of $H$ on $Z^{\prime}$ factors through some $H_{k}$. 
Let $F=Y_{i}$ be an indscheme and $H$ a group-scheme of the above type. An $H$-torsor $\mathcal{H}$ over $F$ is by definition a compatible system of $H_{k}$-torsors $\mathcal{H}_{i, k}$ over the $Y_{i}$ 's. Again, by point (3) of Lemma 8, this notion does not depend on the presentation of $F$ as an inductive limit. For an $H$-torsor $\mathcal{H}$ we could consider its total space, which will be an indscheme not of ind-finite type (unless $H$ is finite dimensional), but this will not be of use for us.

Consider the following situation: let $\mathcal{H}$ be an $H$-torsor over $F$ and let $F^{\prime}$ be another indscheme with a "nice" action of $H$. We claim, that we can form the "associated bundle" $\mathcal{H} \times F^{\prime}$ over $F$, which will be again an indscheme.

Indeed, we can represent $F^{\prime}$ as an inductive limit $Y_{i}^{\prime}$ with each $Y_{k}^{\prime}$ being an $H_{k}$-scheme. We set $\mathcal{H} \stackrel{H}{\times} F^{\prime}$ to be the inductive limit of $\mathcal{H}_{i, i} \stackrel{H_{i}}{\times} Y_{i}^{\prime}$.

A.4. Now we will introduce the category of $H$-equivariant perverse sheaves on an indscheme with a "nice" action of $H$. First, let $Y$ be a scheme of finite type and $H^{\prime}$ an algebraic group acting on it. Then the notion of an $H^{\prime}$-equivariant perverse sheaf on $Y$ is well-known. If $H^{\prime \prime} \rightarrow H^{\prime}$ be a surjection, then the categories $\mathrm{P}_{H^{\prime}}(Y)$ and $\mathrm{P}_{H^{\prime \prime}}(Y)$ are naturally equivalent.

Therefore, if $F=Y_{i}$ with each $Y_{k}$ being $H$-stable and acted on via $H \rightarrow H_{k}$, we can define $\mathrm{P}_{H}(F)$ as $\mathrm{P}_{H_{i}}\left(Y_{i}\right)$.

In particular, let $\mathcal{T}$ be a perverse sheaf on $F, \mathcal{H}$ be an $H$-torsor over $F, F^{\prime}$ be an indscheme with a "nice" $H$-action and, finally, let $\mathcal{S}$ be an $H$-equivariant perverse sheaf on $F^{\prime}$. Then the construction of the twisted external product (cf. Sect. 0.2) makes sense and it produces a perverse sheaf $\mathcal{T} \widetilde{\otimes} \mathcal{S}$ on $\stackrel{H}{*} \times F^{\prime}$.

To introduce the derived categories, we need to make an assumption that $H$ contains a subgroup of finite codimension which is pro-unipotent, i.e. that for large enough $k, H^{k}:=$ $\operatorname{ker}\left(H \rightarrow H_{k}\right)$ is a projective limit of unipotent groups. (This assumption is valid in our examples.)

If $Y$ be a scheme of finite type and $H^{\prime}$ an algebraic group acting on it, the derived category $\mathrm{D}_{H^{\prime}}^{b}(Y)$ has been introduced by Bernstein and Lunts in [7]. Their definition has the following property: if $H^{\prime \prime} \rightarrow H^{\prime}$ is a surjection with a unipotent kernel, then the categories $\mathrm{D}_{H^{\prime \prime}}^{b}(Y)$ and $\mathrm{D}_{H^{\prime \prime}}^{b}(Y)$ are equivalent. This enables us to introduce $\mathrm{D}_{H}^{b}(F)$ : by shifting the indices, we may assume that all the appearing $H^{k}$ 's are already pro-unipotent, and we set $\mathrm{D}_{H}^{b}(F)=\mathrm{D}_{H_{i}}^{b}\left(Y_{i}\right)$.

A.5. Recall our definition of Gr given in Sect. 1.1. Here, for the sake of completeness, we will prove that $\mathrm{Gr}$ is indeed representable by an indscheme. Moreover, from the proof it will follow that any closed subscheme of $\mathrm{Gr}$ is proper over $\mathbb{F}_{q}$. We proceed in two steps:

\section{Proof.}

Step 1. Let us first prove the assertion when $G=G L(n)$. In this case for an affine $S$, $\operatorname{Hom}(S, \mathrm{Gr})$ consists of pairs $\left(\mathcal{V}, \beta^{V}\right)$, where $\mathcal{V}$ is a projective rank $n$ module over $\mathcal{O}_{S}[[t]]$ and $\beta^{V}$ is an isomorphism $\mathcal{V} \underset{\mathbb{F}_{q}[[t]]}{\otimes} \mathbb{F}_{q}((t)) \simeq V \otimes \mathcal{O}_{S}((t))$, where $V$ is the standard $n$-dimensional vector space.

For an integer $m$ consider the $(2 m+1) \cdot n$-dimensional vector space $t^{-m} V[[t]] / t^{m+1} V[[t]]$ and consider the functor that associates to a scheme $S$ the set of all $S$-flat and $t$-stable submodules of $\mathcal{O}_{S} \otimes t^{-m} V[[t]] / t^{m+1} V[[t]]$. This functor is representable by a closed subscheme, call it $\mathrm{Gr}^{m}$, of the Grassmannian of $t^{-m} V[[t]] / t^{m+1} V[[t]]$. In particular, $\mathrm{Gr}^{m}$ is proper. 
We have a natural closed embedding $\mathrm{Gr}^{i} \subset \mathrm{Gr}^{i+1}$, since $t^{-i} V[[t]] / t^{i+1} V[[t]]$ is canonically a $t$-invariant subquotient of $t^{-i-1} V[[t]] / t^{i+2} V[[t]]$. Hence, we obtain a directed family $\mathrm{Gr}^{1} \hookrightarrow$ $\ldots \hookrightarrow \mathrm{Gr}^{i} \hookrightarrow \mathrm{Gr}^{i+1} \hookrightarrow \ldots$, and it is clear that the functor $\mathrm{Gr}$ is isomorphic to $\mathrm{Gr}^{i}$.

Step 2. To treat the case of an arbitrary $G$, it suffices to show that if $G_{1} \rightarrow G_{2}$ is an embedding of reductive groups, then the natural map $\operatorname{Gr}\left(G_{1}\right) \rightarrow \operatorname{Gr}\left(G_{2}\right)$ realizes $\operatorname{Gr}\left(G_{1}\right)$ as a closed subfunctor of $\operatorname{Gr}\left(G_{2}\right)$. For the proof, it will be more convenient to use the realization of the affine Grassmannian via a curve $X$, instead of the formal disc (cf. Lemma 2).

Consider the quotient $G_{2} / G_{1}$. It is a basic fact that under the above circumstances, $G_{2} / G_{1}$ is an affine variety; let $1_{G_{2} / G_{1}} \in G_{2} / G_{1}$ denote the point corresponding to $1 \in G_{2}$.

Thus, let $S$ be a scheme and let $\mathcal{F}_{G_{2}}$ be a $G_{2}$-bundle on $X \times S$, trivialized by means of $\beta$ over $(X \backslash x) \times S$. In particular, we obtain a map

$$
(X \backslash x) \times S \stackrel{\beta\left(1_{G_{2} / G_{1}}\right)}{\longrightarrow} \mathcal{F}_{G_{2}} \stackrel{G_{2}}{\times} G_{2} / G_{1} .
$$

Since $\mathcal{F}_{G_{2}} \stackrel{G_{2}}{\times} G_{2} / G_{1}$ is affine over $X \times S$, there exists a closed subscheme $S^{\prime}$ of $S$, such that for any $\phi: S^{\prime \prime} \rightarrow S$ such that $(X \backslash x) \times S^{\prime \prime} \rightarrow \mathcal{F}_{G_{2}} \stackrel{G_{2}}{\times} G_{2} / G_{1}$ extends to a map $X \times S^{\prime \prime} \rightarrow \underset{\mathcal{F}_{G_{2}}}{G_{2}} \times G_{2} / G_{1}$, $\phi$ factors as $S^{\prime \prime} \rightarrow S^{\prime} \rightarrow S$.

It is easy to see that this $S^{\prime}$ represents the fiber product $S \underset{\operatorname{Gr}\left(G_{2}\right)}{\times} \operatorname{Gr}\left(G_{1}\right)$.

The above argument proves the representability of $\operatorname{Gr}(G)$ for a reductive group $G$. However, $\operatorname{Gr}(G)$ is representable for an arbitrary linear algebraic group:

One shows that if

$$
1 \rightarrow \mathbb{G}_{a} \rightarrow G_{1} \rightarrow G_{2} \rightarrow 1
$$

is a short exact sequence of algebraic groups and $\operatorname{Gr}\left(G_{2}\right)$ is representable, then $\operatorname{Gr}\left(G_{1}\right)$ is represenatble too. For example, when $G_{2}=\{1\}, \operatorname{Gr}\left(\mathbb{G}_{a}\right)$ is isomorphic to the direct limit of linear spaces $t^{-i} \cdot \mathbb{F}_{q}[[t]] / \mathbb{F}_{q}[[t]]$ and the general case is not much different.

However, the above example shows that when $G$ is not reductive, $G r$ is not a limit of proper (i.e. compact) schemes. In general, it is easy to see that for an embedding of algebraic groups $G_{1} \rightarrow G_{2}$, the map $\operatorname{Gr}\left(G_{1}\right) \rightarrow \operatorname{Gr}\left(G_{2}\right)$ is a locally-closed embedding.

A.6. Finally, let us prove Lemma 11. This should be well-known and we include the proof just in order to demonstrate "how things work".

On the one hand, we have the indscheme $\mathcal{G} \stackrel{G(\widehat{\mathcal{O}})}{\times} \operatorname{Gr}$ (cf. Sect. A.3 above) and on the other hand the functor that associates to a scheme $S$ the set of quadruples $\left(\mathcal{F}_{G}, \mathcal{F}_{G}^{1}, \widetilde{\beta}, \beta^{1}\right)$, as in the formulation of the lemma. Both are sheaves on the category of schemes with the étale topology.

Thus, let $\left(\mathcal{F}_{G}^{1}, \beta^{1}\right)$ be an $S$-point of $\mathrm{Gr}$ and we must show that the additional data of $\left(\mathcal{F}_{G}, \widetilde{\beta}\right)$ is equivalent to choosing an $S$-point of $\mathcal{G} \stackrel{G(\widehat{\mathcal{O}})}{\times}$ Gr that projects to our point of $\mathrm{Gr}$.

By making an étale localization, we can assume that $\mathcal{F}_{G}^{1}$ can be trivialized and let us choose such a trivialization. Then, on the one hand, the data of $\left(\mathcal{F}_{G}, \widetilde{\beta}\right)$ becomes equivalent to a data of just another $S$-point of Gr.

Similarly, once $\mathcal{F}_{G}^{1}$ is trivialzed, the induced $G(\widehat{\mathcal{O}})$-torsor over $\mathcal{G} \underset{\mathrm{Gr}}{\times} S$ becomes trivialized too (which means that all the $G(\widehat{\mathcal{O}})_{k}$-torsors become trivialized in a compatible way). Hence $S \underset{\text { Gr }}{\times}(\mathcal{G} \stackrel{G(\widehat{\mathcal{O}})}{\times} \mathrm{Gr})$ splits as $S \times \mathrm{Gr}$. 
This proves the required assertion, once we check that the change of a trivialization of $\mathcal{F}_{G}^{1}$ has the same effect on both sides, which is obvious.

A.7. This subsection is not logically related to anything else in the this appendix. We will reprove the Kazhdan-Lusztig parity vanishing assertion, which was used in the proof of Proposition 1. The argument presented below is presumably well-known:

We will prove a more general fact, namely, the parity vanishing for stalks of IC sheaves of I-orbits on Fl. For an element $\mathrm{w}$ in the affine Weyl group, let $\mathrm{Fl}_{\mathrm{w}}$ denote the corresponding I-orbit in $\mathrm{Fl}$ and $\overline{\mathrm{Fl}}_{\mathrm{w}}$ its closure. Let y be another element with $\mathrm{Fl}_{\mathrm{y}} \subset \overline{\mathrm{Fl}}_{\mathrm{w}}$. We must show that the complex $\left.\mathrm{IC}_{\overline{\mathrm{FI}_{\mathrm{w}}}}\right|_{\mathrm{Fl}_{\mathrm{y}}}$ has cohomology (in either usual or perverse sense) in degrees of constant parity.

In the beginning of the paper we introduced the scheme ConvFl, which was a "two-fold" convolution of $\mathrm{Fl}$ with itself. Along the same lines one introduces the $n$-fold convolution $\mathrm{Conv}_{\mathrm{Fl}}^{n}$. We will denote by $p_{n}$ the natural projection $\mathrm{Conv}_{\mathrm{Fl}}^{n} \rightarrow \mathrm{Fl}$, which generalizes the projection $p$, when $n=2$. If $\mathrm{w}_{1}, \ldots, \mathrm{w}_{n}$ are the $n$ elements of the affine Weyl group, we will denote the corresponding closed subscheme of $\operatorname{Conv}_{\mathrm{Fl}}^{n}$ by $\operatorname{Conv}_{\mathrm{Fl}}^{\mathrm{w}_{1}, \ldots, \mathrm{w}_{n}}$.

Now, let $\mathrm{w}=s_{1} \cdot \ldots \cdot s_{n}$ be a reduced decomposition of $\mathrm{w}$ as a product of simple reflections. Then the restriction of $p_{n}$ to $\operatorname{Conv}_{\mathrm{Fl}}^{s_{1}, \ldots, s_{n}}$ is a proper dominant map onto $\overline{\mathrm{Fl}}_{\mathrm{w}}$. By the decomposition theorem, $\mathrm{IC}_{\overline{\mathrm{FI}_{\mathrm{w}}}}$ is a direct summand of $p_{n !}\left(\mathrm{IC}_{\mathrm{Conv}_{\mathrm{F} 1}^{s_{1}, \ldots, s_{n}}}\right)$. Therefore, it suffices to prove

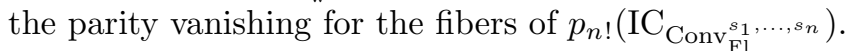

However, since each $\overline{\mathrm{Fl}}_{s_{i}} \simeq \mathbb{P}^{1}$, the variety $\operatorname{Conv}_{\mathrm{Fl}}^{s_{1}, \ldots, s_{n}}$ is non-singular (it is commonly referred to as the Bott-Samelson resolution of $\overline{\mathrm{Fl}}_{\mathrm{W}}$ ), hence, $\mathrm{IC}_{\mathrm{Conv}_{\mathrm{F} 1}^{s_{1}, \ldots, s_{n}}}$ is the constant sheaf, up to a cohomological shift. By base change, it therefore suffices to show that the fiber of $\operatorname{Conv}_{\mathrm{Fl}}^{s_{1}, \ldots, s_{n}}$ over every given point in $\overline{\mathrm{Fl}}_{\mathrm{y}}$ has cohomology in degrees of constant parity.

Now, it is known (and easily proven by induction) that each such fiber, call it $Y$, can be represented as is a union on locally closed subvarieties $Y_{i}$, each of which is isomorphic to a tower of affine spaces. Therefore, the Cousin spectral sequence implies that $H_{c}(Y)$ lives only in even degrees.

\section{REFERENCES}

[1] A. Beilinson, How to glue perverse sheaves, in: Lectures notes in Mathematics, 1289, (1987), pp. 42-51.

[2] A. Beilinson, J. Bernstein, A proof of Jantzen conjectures, Adv. in Sov. Math 16 (1993), pp. 1-50.

[3] A. Beilinson, J. Bernstein and P. Deligne, Faisceaux pervers, Astérisque 100 (1982), pp. 5-171.

[4] A. Beilinson, V. Drinfeld, Quantization of Hitchin's Hamiltonians and Hecke eigensheaves, preprint.

[5] A. Beauville, Y. Laszlo, Un lemme de descente, C.R. Acad. Sci. Paris, Sér. I Math. 320 (1995), pp. 335-340.

[6] J. Bernstein, Le "centre" de Bernstein, Travaux en Cours, Representations of reductive groups over local fields (1984), pp. 1-32.

[7] J. Bernstein, V. Luntz, Equivariant sheaves and functors, Lecture notes in Math. 1578 (1994).

[8] R. Bezrukavnikov, private communications.

[9] V. Drinfeld, C. Simpson B-structures on G-bundles and local triviality, Math. Res. Lett 2 (1995), pp. 823-829.

[10] A. Grothendieck, Appendix to Good reduction of abelian varieties by Serre and Tate, Ann. of Math. 88 (1968), pp. 514-516.

[11] E. Frenkel, D. Gaitsgory and K. Vilonen Whittaker patterns in the geometry of moduli spaces of bundles on curves, preprint alg-geom/9907133.

[12] D. Kazhdan and G. Lusztig Schubert varieties and Poincaré duality, Proc. Symp. Pure Math. XXXVI (1980), pp. 185-203.

[13] G. Lusztig, Singularities, character formulas, and a q-analogue of weight multiplicities, Astérisque 101 (1983), pp. 208-229.

[14] I. Mirkovic and K. Vilonen, Perverse sheaves on affine Grassmannians and Langlands duality, preprint alg-geom/9911050.

[15] SGA 7, volume II, Exposé XIII. 
D. GAITSGORY

Department of Mathematics, Harvard University, Cambridge MA 02138

E-mail address: gaitsgde@math.harvard.edu 